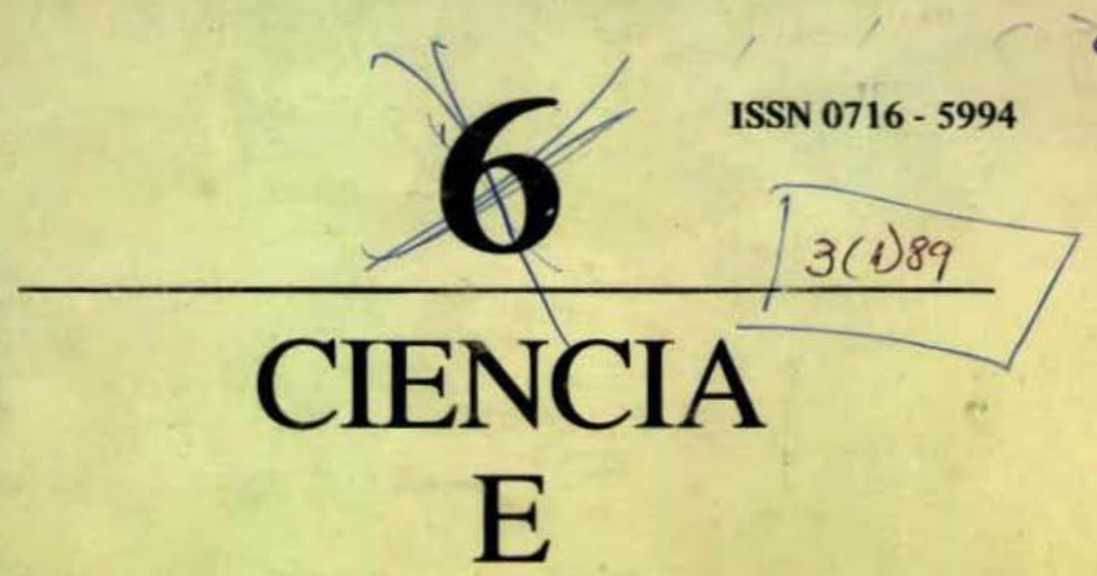

\title{
INVESTIGACION FORESTAL
}

JUNIO 1989

Iif 90 CHILE

FILIAL CORFO 


\title{
RENOVALES DE RAULI, ROBLE, COIGUE Y TEPA EXPECTATIVAS DE RENDIMIENTO
}

(Charla aportada al Simposio "Silvicultura de los tipos forestales de la región Centro-Sur de Chile" realizada en la Universidad Austral en Valdivia del 27.03.89 - 07.04.89)

Hans Grosse W.*

\begin{abstract}
RESUMEN
En la Precordillera Andina de la X Región (49 $45^{\prime}$ ' Lat. Sur sector Neltume) se realizaron estudios para establecer el rendimiento de renovales de raulí, roble, coiguie y tepa.

Con la información recopilada y procesada a través de análisis de tallo fue posible obtener cifras que orientan sobre el crecimiento diametral de las especies en estudio en función de sus variables de estado, de los árboles competidores más directos y del espacio disponible para su desarrollo.

Para distintas situaciones de renoval tipo de la zona, se entregan a modo de ejemplo esquemas de intervención silvícola y la proyección del renoval en el tiempo.

Palabra clave: Rendimiento, renovales, silvicultura.
\end{abstract}

\begin{abstract}
Second growth stand data of Rauli, Roble, Coigüe and Tepa, located in the Andean foothills of the 10th Region of Chile (Lat. 49' 45' S) were analized to construct individual species yield models.

The results of stem analyses were modeled to include the effect of the nearest competitor and the total space available for development.

The results were used to construct models to estimate what volumetric changes could occur if a certain silvicultural practice was applied.
\end{abstract}

(*) Ingeniero Forestal, Dr. División Regional. Barros Arana 121, Concepción. Chile. 


\section{INTRODUCCION}

Durante los últimos af́os han llamado la atención los renovales constituidos por especik nativas, como unidades con un potencial altamente rentable. Dentro de éstas situaciones he destacado aquellos rodales con presencia de árboles del género Nothofagus. El gran número $v$ individuos, que generalmente se encuenta en estos bosques jóvenes, hacen necesario raleos. q en su mayoría son de carácter comercial. La apertura del mercado de astillas para la fibra corte significa una buena posibilidad para manejar este recurso en términos rentables valorizando la individuos remanentes. Los renovales constituyen un mosaico de situaciones muy heterogénec. fundamentado en la existencia de distintas especies y diferentes estados de desarrollo y comp: tencia.

Esto implica que las decisiones técnicas a tomar no podrán ser rígidas, sino que deberí adaptarse a cada situación en especial. En consecuencia, deberán prepararse equipos técnicu capaces de elaborar pautas silvícolas y de aplicarlas eficientemente.

Los tipos forestales a los que pertenecen las unidades de muestreo son "Roble - Rauli. Coigue" y "Coigüe - Raulf - Tepa". Los ejemplos que se entregan fueron construidos sobre h base de situaciones reales.

El objetivo del presente trabajo es el de analizar aplicaciones de modelos silvícolas consi derando situaciones monoespecíficas y con la participación de varias especies para renovales er distinto estado de desarrollo. Para éstos se aplican esquemas de raleo y cosecha durante el peric. do de manejo.

\section{ANTECEDENTES METODOLOGICOS}

La información recopilada proviene de parcelas temporales. Estas se instalaron con el objetivo de conocer el desarrollo de las variables de estado de los rodales y construir modelos de crecimiento para distintos estados de desarollo y competencia.

Antecedentes metodológicos referidos al muestreo para el análisis de los árboles individua les, como la construcción de modelos de crecimiento diametral sólo se entregan en forma resu. mida, para una mejor comprensión inmediata por parte del lector. Una descripción más ampliade éstos se encuentra en publicaciones anteriores, mencionadas en el texto.

Los modelos de crecimiento diametral, DAP - volumen y DAP - altura también se entre. gan en el presente capítulo, entendiéndose como herramientas básicas para la descripción y pro. yección de los rodales.

\section{Descripción de las parcelas muestreadas}

\section{Parcelas con predominancia de rault}

Las parcelas temporales con predominancia de raulí se localizaron en el área de Neltume en los sectores denominados "Los Hornos - Pidihuil", "Pto. Fui" y "Quebrada Honda" (Precordillera Andina, $39^{\circ} 45^{\prime}$ Lat. Sur, comuna de Panguipulli, provincia de Valdivia, X Región) (GROSSE y CUBILLOS, 1987 y CUBILLOS, 1987). Las parcelas se plantearon en $25 \mathrm{~m}$ x $25 \mathrm{~m}$ $\left(625 \mathrm{~m}^{2}\right)$.

En cada parcela se midieron todos los árboles con un DAP igual o mayor a $5 \mathrm{~cm}$. Además se midieron todos los individuos fuera de la parcela, que proyectaban su copa hacia el interior de ésta. 
Los individuos se localizaron espacialmente.

Las variables de estado medidas en cada individuo fueron:

- Diámetro a la altura del pecho (DAP o $1,3 \mathrm{~m}$ )

- Altura total (m)

- Altura de inicio de copa (m)

- Radios de copa, medidos en dirección de los cuatro puntos cardinales (m)

Las parcelas con predominancia de roble, coigüe y tepa

Las parcelas temporales con predominancia de roble, coigüe y tepa también se localizaron en el área de Neltume. Los sectores específicos por especie fueron: roble (Los Hornos, Pto. Fuy), coigüe (Los Hornos - Pidihuil, Truful), Tepa (Huilo - Huilo, Molco) (GROSSE et al., 1988; CUBILLOS, 1988). Las unidades de muestreo fueron parcelas de $500 \mathrm{~m}^{2}$, instaladas en sectores representativos para cada situación de renoval en análisis.

Las variables de estado medidas para éstas tres especies fueron las mismas que en las parcelas de raulí.

\section{Muestreo para el análisis de árboles individuales}

Para realizar el análisis del árbol individual o árbol sujeto, para cada especie se seleccionó una amplia muestra de individuos representativa del rango de edad, altura y del espaciamiento encontrado en cada situación del renoval. Una vez identificados los "árboles sujetos" se ubicaron y registraron los árboles que ejercían la competencia más directa sobre éstos. (GROSSE y CUBILLOS, 1987; CUBILLOS, 1987; GROSSE et. al, 1988; CUBILLOS, 1988).

La información recopilada en terreno para los árboles sujeto y sus competidores fue:

- Diámetro a la altura del pecho (DAP a 1,3 m)

- Altura total

- Altura de inicio de copa

- Radios de copa, medidos en dirección de los cuatro puntos cardinales

- Distancias entre el árbol sujeto y sus competidores.

Luego se voltearon los árboles sujeto, seccionándolos en trozas de $2 \mathrm{~m}$. De cada una de éstas secciones y a la altura del DAP, se extrajeron rodelas para su medición en laboratorio.

Modelos de crecimiento diametral

Los árboles sujeto fueron sometidos a análisis de tallo utilizando el programa ANATAL (ALVAREZ, 1977). Esto dio origen a una serie de antecedentes dasométricos básicos con los cuales se construyeron modelos de crecimiento diametral (DAP), (GROSSE y CUBILLOS, 1987; CUBILLOS, 1987; GROSSE et. al, 1988; CUBILLOS, 1988; GROSSE, 1989).

Estos consideran los últimos cinco affos de vida del árbol, suponiendo una situación de competencia similar a la encontrada en el momento del muestreo.

Las variables incorporadas a los modelos de crecimientn diametral (IDAPA) utilizados en este estudio son:

E : Edad (años)

DAP : Diámetro a la altura del pecho (a $1,3 \mathrm{~m}$ de altura en $\mathrm{cm}$ )

DC : Diámetro de copa (m)

AC : Cobertura de copa del árbol $\left(\mathrm{m}^{2}\right)$

H. TOT. : Altura total (m)

HINIC : Altura de inicio de la copa $(\mathrm{m})$

HINICCO : Altura de inicio de copa de los competidores (m)

DISTME : Distancia media entre el árbol sujeto y sus competidores (m) 
Los modelos de crecimiento diametral son:

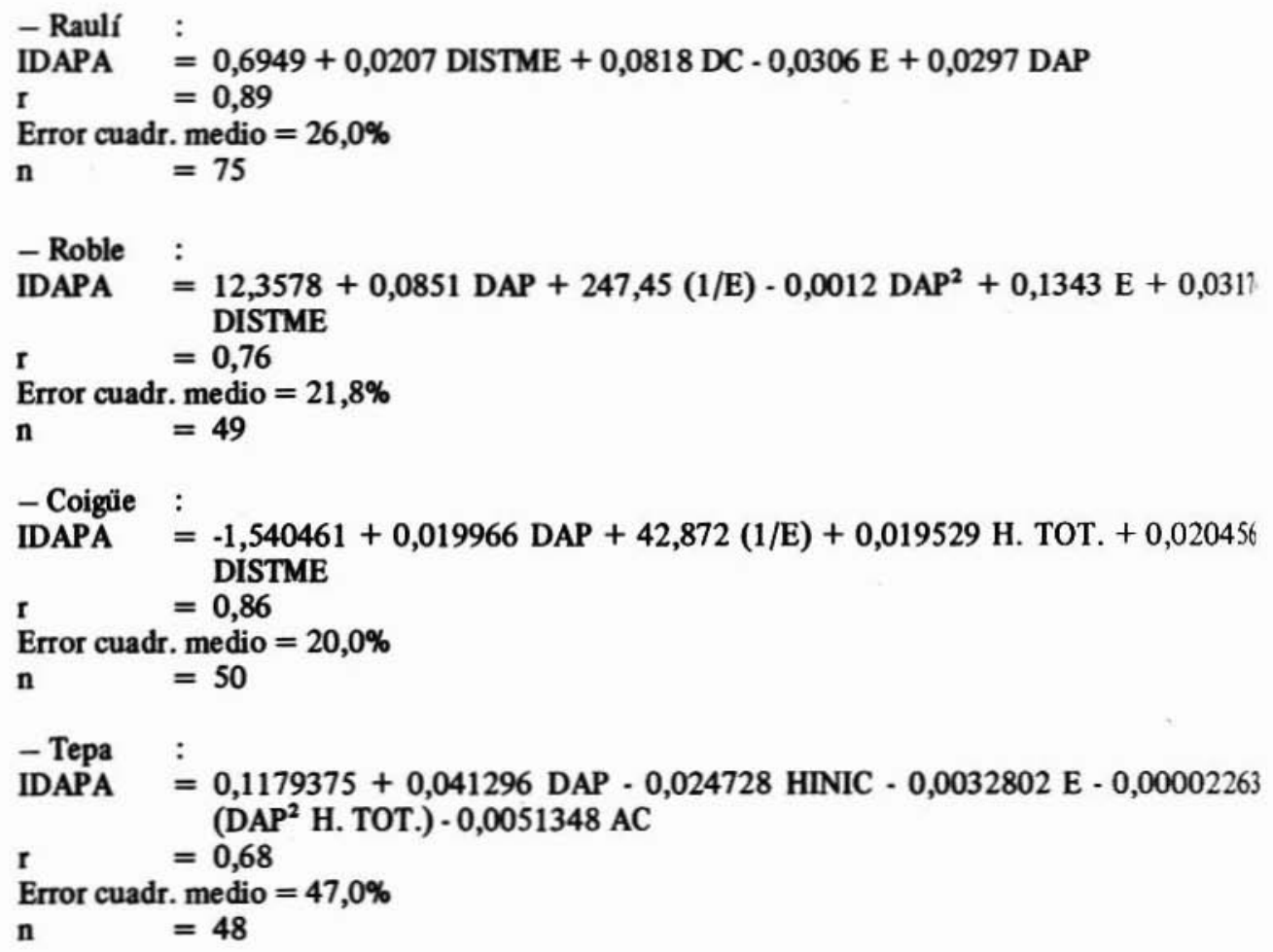

\section{Proyección de rodales}

El análisis de intervenciones planteadas para cada una de las parcelas temporales se realizó en base a las tablas de rodal, de área basal y la distribución espacial de los individuos.

La proyección de crecimiento se realizó sobre la base del incremento diamétrico, utilizan. do las funciones de incremento diamétrico presentadas anteriormente. Esta se realizó hasta las edades que limitan con la capacidad de proyección de los modelos.

Los volúmenes fueron estimados con las siguientes regresiones, construidas para el área de Neltume (CUBILLOS, 1988 a,b; GROSSE et. al, 1988).

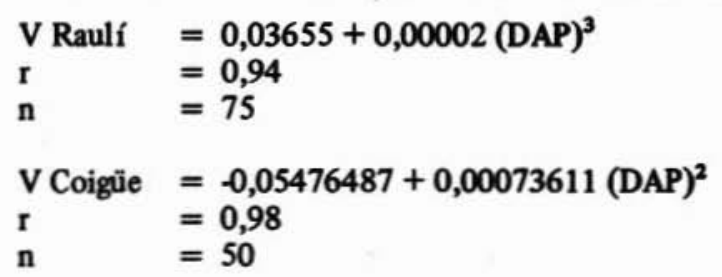


HANS GROSSE W.

$$
\begin{array}{lll}
\text { V Roble } & =-0,03695309+0,00075407(\text { DAP })^{2} \\
\mathrm{r} & =0,98 \\
\mathrm{n} & =50 \\
& & \\
\text { V Tepa } & =-0,04220197+0,00067576(\mathrm{DAP})^{2} \\
\mathrm{r} & =0,97 \\
\mathrm{n} & =50
\end{array}
$$

Donde: $\quad \mathrm{V}=$ Volumen en $\mathrm{m}^{3} \mathrm{ssc}$

DAP $=$ Diámetro a la altura del pecho $(\mathrm{cm})$

Las regresiones DAP - altura por especie y rodal seleccionado son:

$$
\begin{aligned}
\text { H Rauli (Los Hornos) } & =-3,6163+8,6401 \text { In DAP } \\
\mathrm{r} & =0,79 \\
\mathrm{n} & =84
\end{aligned}
$$

H Raulí (Q. Honda) $=-0,6920+5,4465$ In DAP

$$
\begin{array}{ll}
\mathrm{r} & =0,74 \\
\mathrm{n} & =21
\end{array}
$$

$$
\begin{aligned}
\text { H Roble (Los Hornos) } & =-2,1307+7,6215 \text { In DAP } \\
\mathrm{r} & =0,83 \\
\mathrm{n} & =52
\end{aligned}
$$

H Roble (Fuy) $\quad=12,0311+0,2792$ DAP

$$
\begin{array}{ll}
\mathbf{r} & =0,94 \\
\mathbf{n} & =22
\end{array}
$$

$$
\begin{aligned}
\mathrm{H} \text { Coigüe (Truful) } & =-0,4158+6,1591 \text { In DAP } \\
\mathrm{r} & =0,83 \\
\mathrm{n} & =81
\end{aligned}
$$

$\mathrm{H}$ Coigüe (Pidihuil) $=9,6594+0,8424 \mathrm{DAP}-0,0084 \mathrm{D}^{2}$

$$
\begin{array}{ll}
\mathrm{r} & =0,86 \\
\mathrm{n} & =22
\end{array}
$$

H Tepa (Molco) $\begin{aligned} & =-3,0712+6,6982 \text { In DAP } \\ \mathrm{r} & =0,84 \\ \mathrm{n} & =156\end{aligned}$

$$
\text { H Tepa (Huilo) } \begin{aligned}
& =4,3204+0,5017 \text { DAP } \\
\mathrm{r} & =0,94 \\
\mathrm{n} & =35
\end{aligned}
$$

Donde: $\quad \mathrm{H}=$ Altura en $\mathrm{m}$

DAP $=$ Diámetro a la altura del pecho $(\mathrm{cm})$ 


\section{RESULTADOS Y DISCUSION}

A continuación se proponen y comentan diferentes tipos de intervención para renovales del área de Neltume.

El crecimiento de renovales de raulí

Los rodales elegidos para raulí presentan dos situaciones:

- Rodal de 36 años sin intervención anterior

- Rodal de 24 años con raleo suave, diez años antes de realizar la medición.

Proyección de un renoval sin intervención anterior (Edad: 36 años)

El rodal sin intervención está constituido por 1.728 árboles por ha. La participación ma. yoritaria de las especies, en términos numéricos se concentra en rauli y roble con un $78 \%$ y un $5 \%$ respectivamente. El resto está constituido por coigües, tepas y trevos (Figura 1). El área basal es de aproximadamente $53 \mathrm{~m}^{2} / \mathrm{ha}$, la que se consideró como la capacidad de ocupación del sitio para las especies en cuestión, a los 36 años.

Los valores de altura - DAP indican una participación de árboles sobre $11 \mathrm{~cm}$ de DAP. en los estratos codominante y dominantes. Los individuos con diámetros menores pertenecen al estrato intermedio y suprimido (Figura 2).

\section{FIGURA 1 \\ PROYECCION DE COPAS - RENOVAL SIN INTERVENCION PREVIA EDAD 36 AÑOS (SECTOR: LOS HORNOS - PIDIHUIL)}

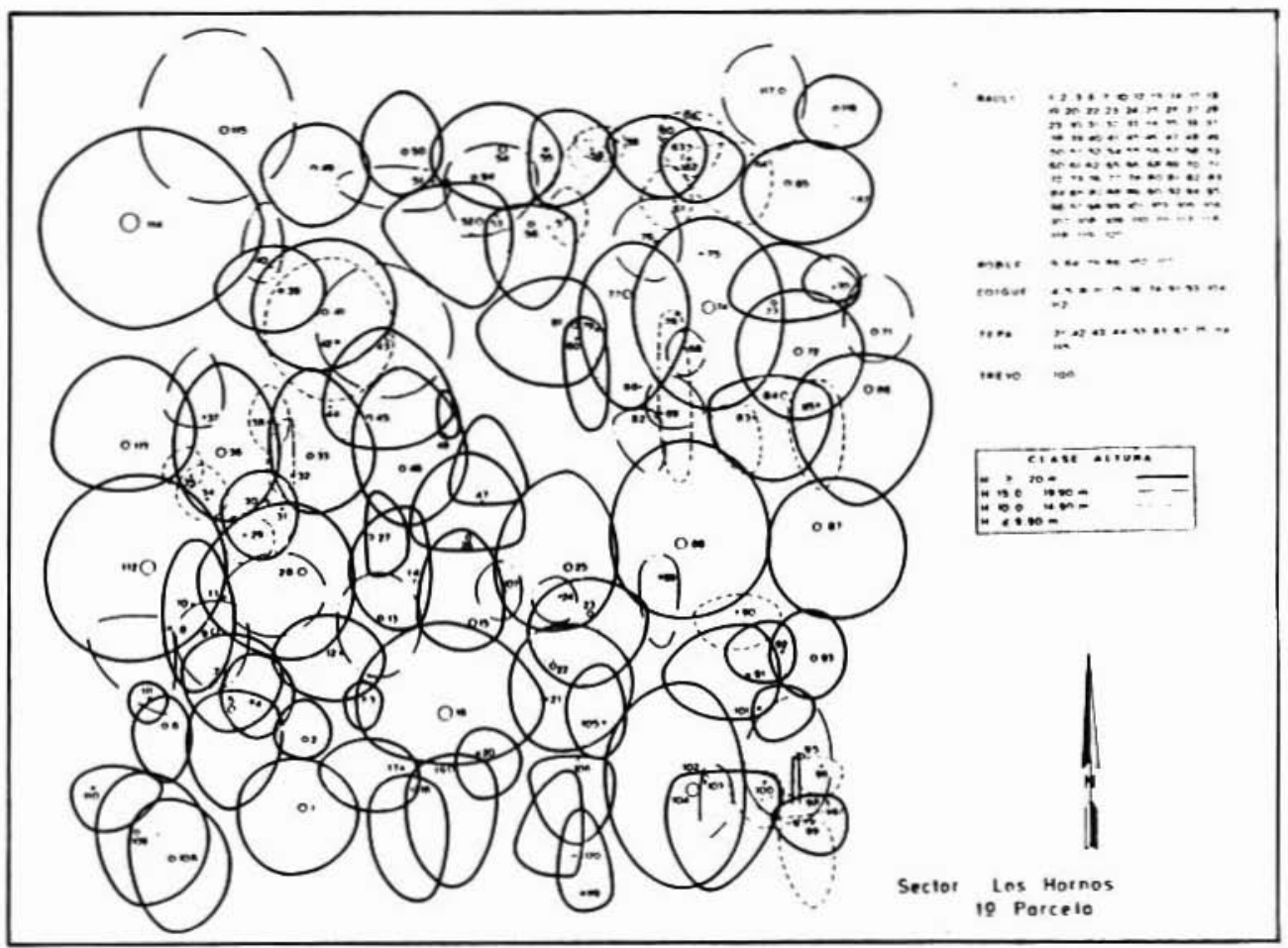


La tabla de rodal se clasificó en cuatro rangos, correspondiendo el $\mathrm{A}$ a los individuos suprimidos e intermedios, el $\mathrm{B}$ a los intermedios a dominantes, el $\mathrm{C}$ a los codominantes y dominantes y el D a los dominantes que podrían ser calificados como "árboles lobos" o pertenecientes a la generación anterior (Figura 2).

El raleo tiene como objetivo principal el de liberar aquellos árboles que se agrupan entre las clases diamétricas 20 a 34 . Corresponden a individuos del estrato dominante y codominante (C), con la mejor posibilidad de producir un alto volumen aserrable en el corto plazo (Cuadro 1). Sus competidores deberán eliminarse a través de un raleo por lo alto o anillamiento. Sus competidores se localizan dentro del sector $\mathrm{C}$ y en las clases diamétricas inmediatamente inferiores $\mathrm{y}$ superiores. La eliminación de éstos contempla la extracción de coigües, trevos y tepas dentro del rango $\mathrm{C}, \mathrm{y}$ raleos por lo alto $\mathrm{y}$ anillamientos fuera de éste.

El área basal a extraer corresponde a un $45 \%$ del área basal del estrato dominante y codominante $(B+C+D)$. El estrato suprimido e intermedio (A), no se consideró para el cálculo de este porcentaje, por su poca importancia en su rol como competidor, dado que las condiciones de sitio de los rodales en estudio no presentan limitaciones de agua y difícilmente de nutrientes.

La relación altura/diámetro de los árboles remanentes bordea el valor 0,97. Este indicador muestra que con la intervención propuesta el rodal de raulí-roble mantiene características de estabilidad suficientes, para no correr riesgos mayores por vientos fuertes, considerando de que se trata de especies de hoja caduca.

\section{CUADRO 1}

INTERVENCION AL RENOVAL - AÑO 0 (RAULI)

(Edad: 36 Años, Sector Los Homos)

\begin{tabular}{|l|c|c|c|c|}
\hline \multicolumn{1}{|c|}{$\begin{array}{c}\text { Situac. después de la } \\
\text { intervención }\end{array}$} & Rango & $\begin{array}{c}\text { Area basal } \\
\mathbf{m}^{2} / \mathbf{h a}\end{array}$ & $\begin{array}{c}\% \text { Area basal } \\
\text { relac. a B+C+D }\end{array}$ & N/ha \\
\hline Estrato Domin. y Codom. & B+C+ D & 50,0 & 100,0 & 1.232 \\
$\begin{array}{l}\text { Estrato a extraer } \\
\text { B+ D+ (C*) }\end{array}$ & 22,4 & 44,8 & 672 \\
$\begin{array}{l}\text { Estrato reman. } \\
\text { (Dom y Cod.) }\end{array}$ & $\begin{array}{c}\text { C (Rauli + } \\
\text { Roble) }\end{array}$ & 27,5 & 55,0 & 560 \\
$\begin{array}{l}\text { Estrato reman. (Interm. + } \\
\text { Suprim.) }\end{array}$ & A & 2,9 & & 496 \\
Total & & 52,9 & & \\
\hline
\end{tabular}

$C^{*}$ : Otros: Coigües, Tepas, Trevos. 
FIGURA 2 A, B, C

\section{ESTADO DEL RENOVAL AL AÑO 0}

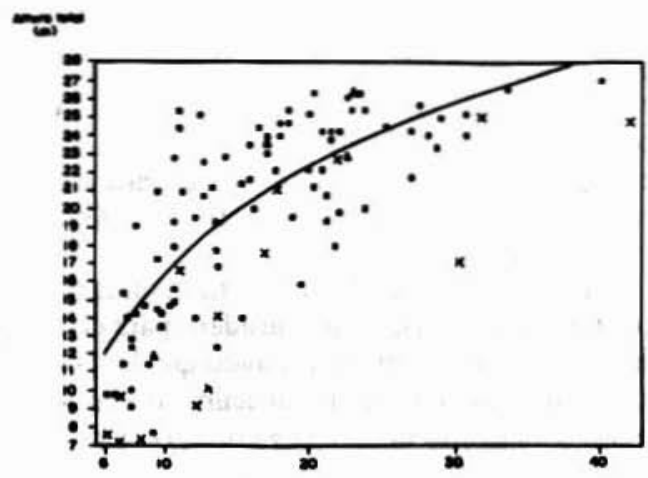

(Especie : Rauli)

(Edad : 36 años)

(Sector : Los Hornos)

A : Altura Total (m) - DAP

B : Area Basal $\left(\mathrm{m}^{2}\right) \cdot$ DAP

C : TABLA DE RODAL

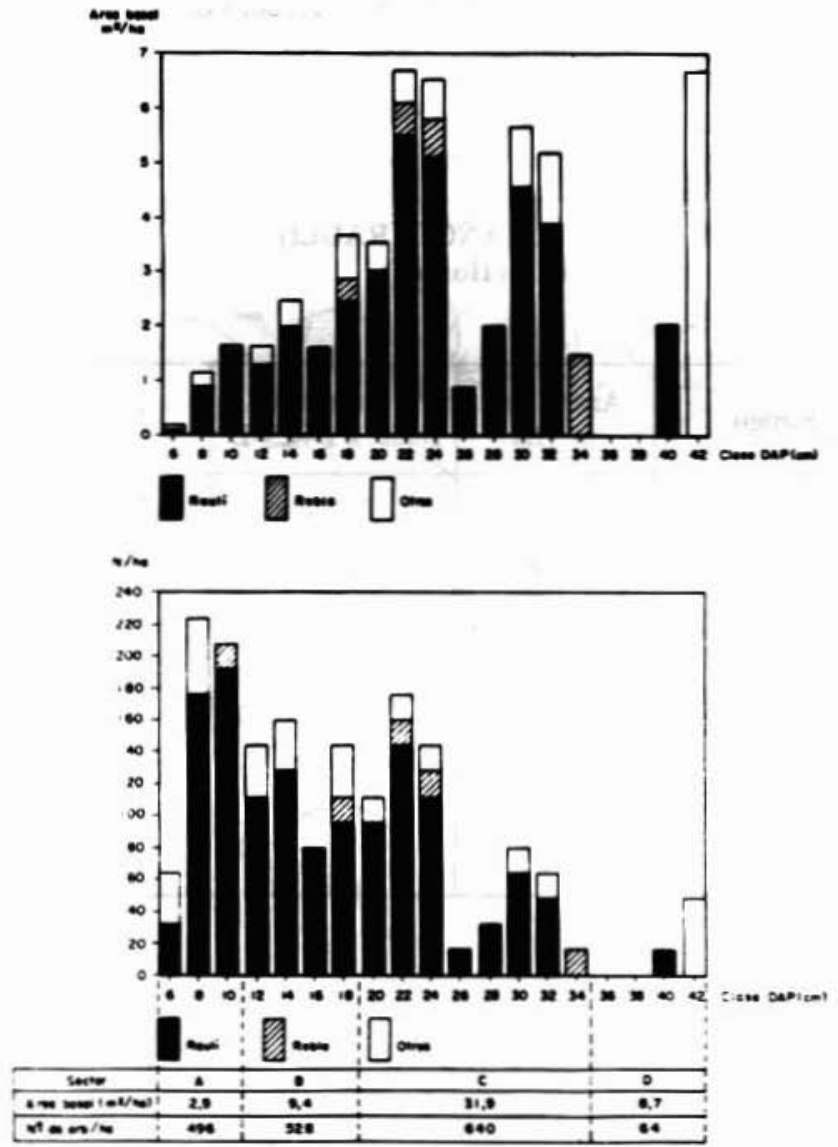

44 / Ciencia e Investigación Forestal 


\section{CUADRO 2 \\ PROYECCION RAULI \\ Sector Los Hornos (sin intervención previa)}

\begin{tabular}{|c|c|c|c|c|c|c|}
\hline \multicolumn{2}{|l|}{ Año } & $\mathbf{0}$ & 5 & 10 & 15 & 20 \\
\hline \multicolumn{7}{|c|}{ RAULI } \\
\hline $\begin{array}{l}\overline{\mathrm{DAP}}(\mathrm{cm}) \\
\mathrm{E} \\
\mathrm{DC}(\mathrm{m}) \\
\text { DISTME }( \\
\mathrm{AB}\left(\mathrm{m}^{2} / \mathrm{h}\right. \\
\mathrm{N} / \mathrm{ha} \\
\text { IDAPA (c }\end{array}$ & & $\begin{array}{c}24,8 \\
36 \\
4,2 \\
4,2 \\
24,7 \\
512 \\
0,76\end{array}$ & $\begin{array}{c}28,6 \\
41 \\
4,2 \\
4,2 \\
32,9 \\
512 \\
0,72\end{array}$ & $\begin{array}{c}32,2 \\
46 \\
* \quad 4,7 \\
* \quad 4,7 \\
36,7 \\
* 450 \\
0,73\end{array}$ & $\begin{array}{c}35,8 \\
51 \\
4,7 \\
4,7 \\
45,3 \\
450 \\
0,68\end{array}$ & $\begin{array}{r}39,2 ! \\
56 \\
4,7 \\
4,7 \\
54,3 \\
450 \\
\ldots\end{array}$ \\
\hline \multicolumn{7}{|c|}{ ROBLE } \\
\hline $\begin{array}{l}\overline{\mathrm{DAP}}(\mathrm{cm}) \\
\mathrm{E} \\
\text { DISTME }( \\
\mathrm{AB}\left(\mathrm{m}^{2} / \mathrm{h}\right. \\
\mathrm{N} / \mathrm{ha} \\
\text { IDAPA }\end{array}$ & & $\begin{array}{c}26,7 \\
36 \\
4,2 \\
2,7 \\
48 \\
0,89\end{array}$ & $\begin{array}{c}31,2 \\
41 \\
4,2 \\
3,7 \\
48 \\
0,79\end{array}$ & $\begin{array}{c}35,2 \\
46 \\
* \quad 4,7 \\
* \quad 4,7 \\
* \quad 48 \\
\quad 0,83\end{array}$ & & \\
\hline \multicolumn{7}{|c|}{ A NIVEL DE RODAL (RAULI + ROBLE) } \\
\hline $\begin{array}{l}\text { DISTME } \\
\mathrm{AB}\left(\mathrm{m}^{2} / \mathrm{h}\right. \\
\mathrm{N} / \mathrm{ha}\end{array}$ & & $\begin{array}{r}4,2 \\
27,4 \\
560\end{array}$ & $\begin{array}{r}4,2 \\
36,6 \\
560\end{array}$ & $\begin{array}{r}4,7 \\
46,4 \\
* 450\end{array}$ & $\begin{array}{r}4,7 \\
45,3 \\
450 \\
\hdashline \\
\hdashline\end{array}$ & $\begin{array}{r}4,7 \\
54,3 \\
450 \\
\end{array}$ \\
\hline \multicolumn{7}{|c|}{ VOLUMEN $\left(\mathrm{m}^{3} / \mathrm{ha}\right)$} \\
\hline Remanent & $\begin{array}{l}\text { e Ra. } \\
\text { Ro. }\end{array}$ & $\begin{array}{r}175 \\
24\end{array}$ & $\begin{array}{r}258 \\
34\end{array}$ & 317 & 430 & 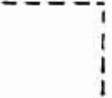 \\
\hline A extraer & $\begin{array}{l}\text { Ra. } \\
\text { Ro. } \\
\text { Otros } 190\end{array}$ & 190 & & $\begin{array}{l}44 \\
44\end{array}$ & 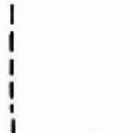 & 559 \\
\hline$\Sigma$ a extrae & & 190 & & 88 & in & \begin{tabular}{r|r}
559 \\
\hdashline
\end{tabular} \\
\hline
\end{tabular}

* : A los 10 años se extraen 48 robles $(\overline{\mathrm{DAP}}=35,2)$ y 62 raulíes $(\overline{\mathrm{DAP}}=32,2)$

: Valores extrapolados

Otros: Coigüe, Tepa, Trevo y Rauli de la clase $\overline{\mathrm{DAP}} 40$. 
La proyección del rodal presentada en el cuadro 2, sólo incorpora raulíes y robles. Los coigües, tepas y trevos serán cortados junto a los árboles lobos de raulí al inicio del período. El volumen a extraer al año 0 corresponde a $190 \mathrm{~m}^{3} / \mathrm{ha}$. La segunda intervención se realiza al año 10 , extrayéndose $88 \mathrm{~m}^{3}$ de raulíes y robles (DAP 32,2 y 35,2 respectivamente), quedando como remanentes sólo raulíes. Estos se explotan al afio 20 con un diámetro medio aproximado de $40 \mathrm{~cm}$ obteniéndose un volumen de $559 \mathrm{~m}^{3} / \mathrm{ha}$.

El volumen total a extraer en el período de 20 affos es de $837 \mathrm{~m}^{3} /$ ha, de los cuales 278 $\mathrm{m}^{3}$ /ha corresponden a raleos.

\section{Proyección de un renoval con intervención anterior (Edad: 24 años)}

El rodal con intervención anterior presenta 672 árboles por ha a los 24 años. De éstos el $50 \%$ está constituido por raulí y el resto por tepa y trevo (Figura 3). Su área basal se acerca a $\operatorname{los} 18 \mathrm{~m}^{2} /$ ha (Figura 4).

\section{FIGURA 3}

\section{PROYECCION DE COPAS - RENOVAL CON INTERVENCION PREVIA EDAD: 24 AÑOS (SECTOR QUEBRADA HONDA)}

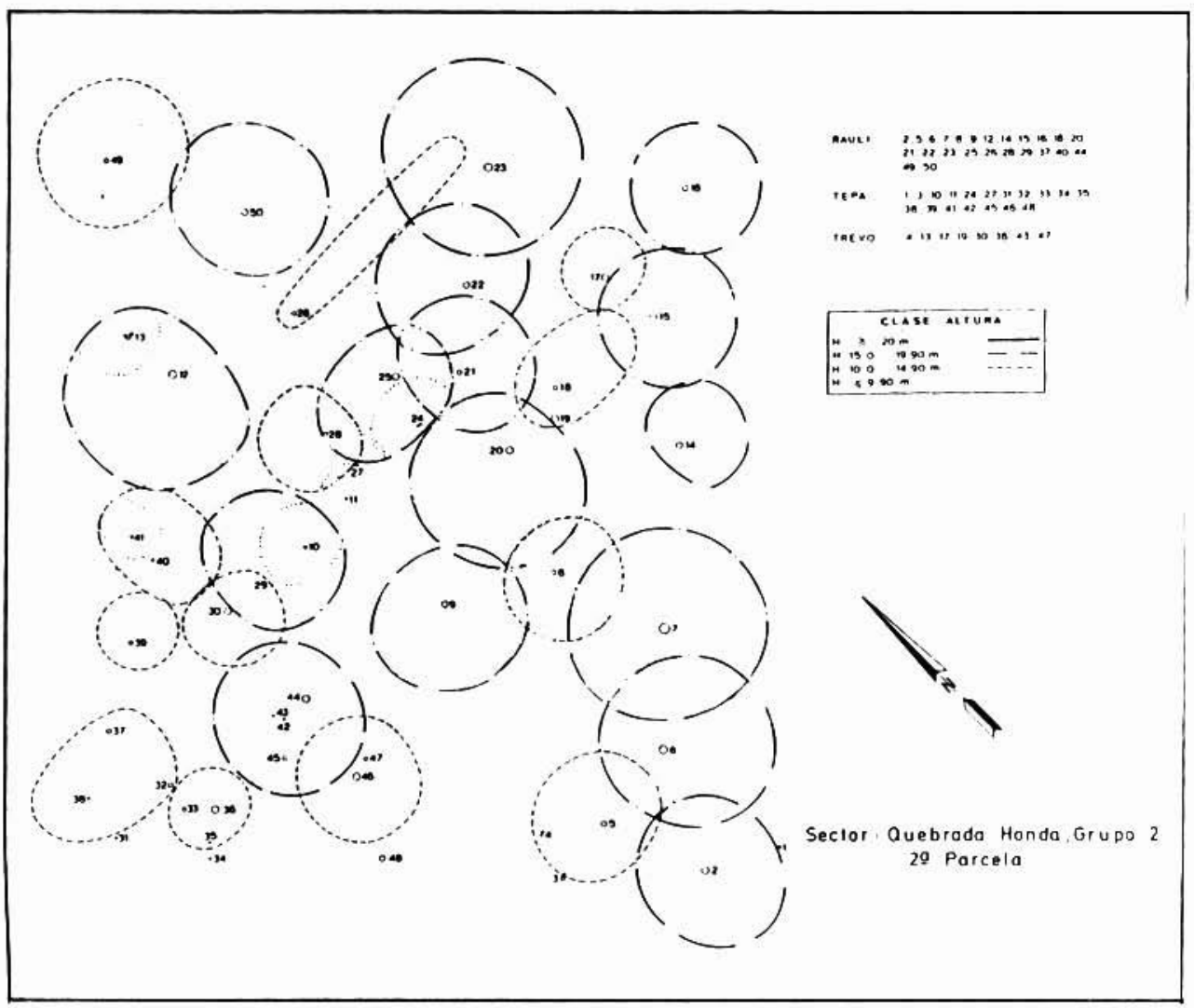


HANS GROSSE W.

FIGURA 4 A, B, C

ESTADO DEL RENOVAL AL AÑO 0

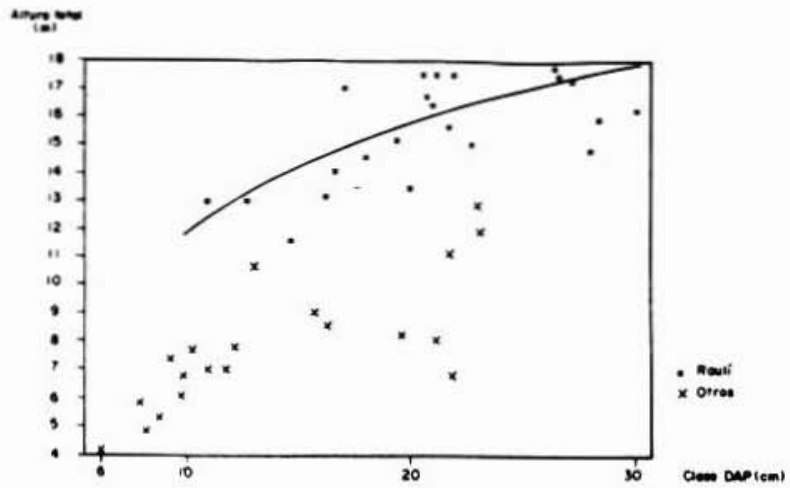

(Especie : Rauli)

(Edad : 24 años)

(Sector : Quebrada Honda)
A : Altura Total (m) - DAP
B : Area Basal $\left(\mathrm{m}^{2}\right)$ - DAP
C : Tabla de Rodal
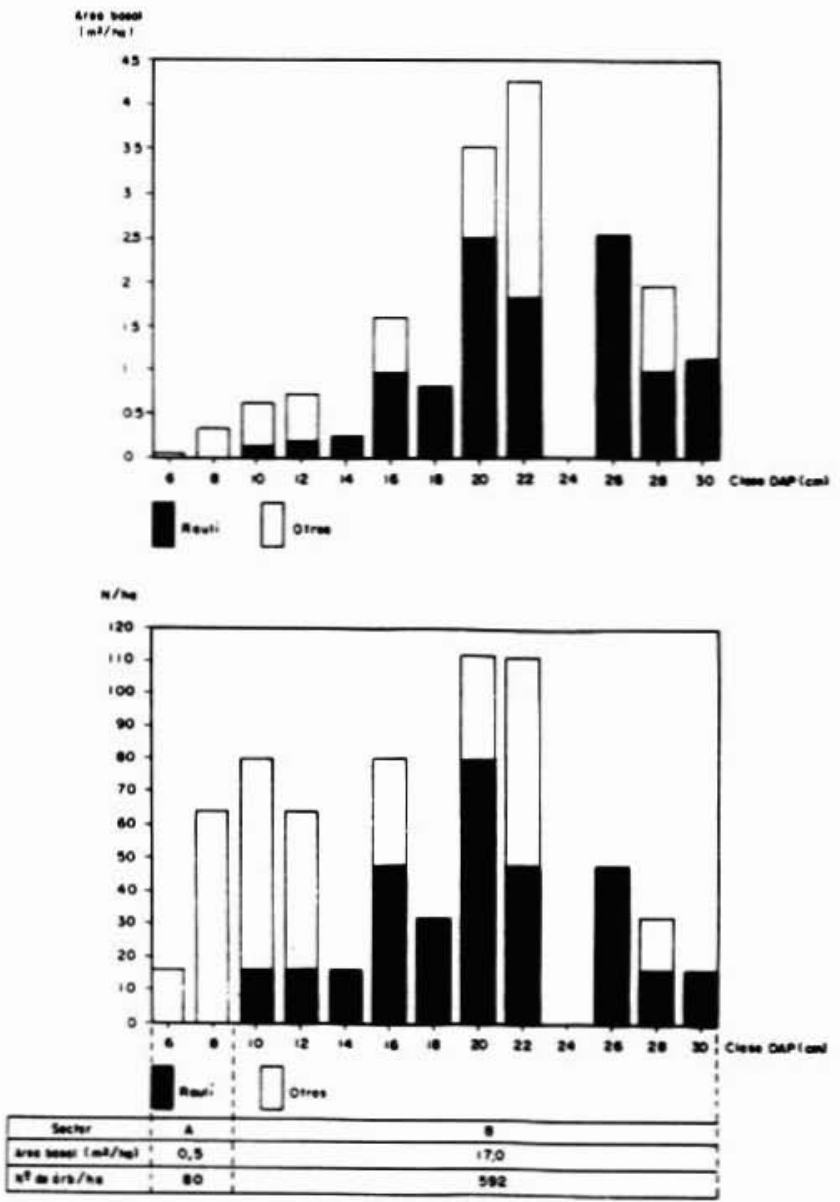
En este caso se proyecta la totalidad del raulí del estrato codominante y dominante (B), es decir, 336 individuos entre las clases 10 y $30 \mathrm{~cm}$ de DAP, que supuestamente llegarán al final de la rotación (Figura 4).

$\mathrm{Al}$ af́o $\mathbf{0} \mathrm{no}$ se interviene el rodal debido al bajo número de individuos. No se considera necesario ni recomendable incrementar el número de individuos a través de una plantación, por considerarse como suficiente el número de árboles existentes (Cuadro 3).

El primer raleo se realiza a los 5 affos, el segundo a los 15 años y el último a los 25 años. El volumen total a extraer en raleos es de $75 \mathrm{~m}^{3} / \mathrm{ha}$. La explotación final al cumplirse un perío. do de 30 ańos considera $925 \mathrm{~m}^{3} / \mathrm{ha}$ de raulí. La producción total es de $1.000 \mathrm{~m}^{3} / \mathrm{ha}$.

Este tipo de manejo permite un crecimiento diametral sostenido promedio de $1 \mathrm{~cm}$ o más. Esto permite que en un periodo de manejo de 20 años, el diámetro medio de los raulies pase los $50 \mathrm{~cm}$.

\section{CUADRO 3}

PROYECCION RAULI

Sector Quebrada Honda (con intervención previa)

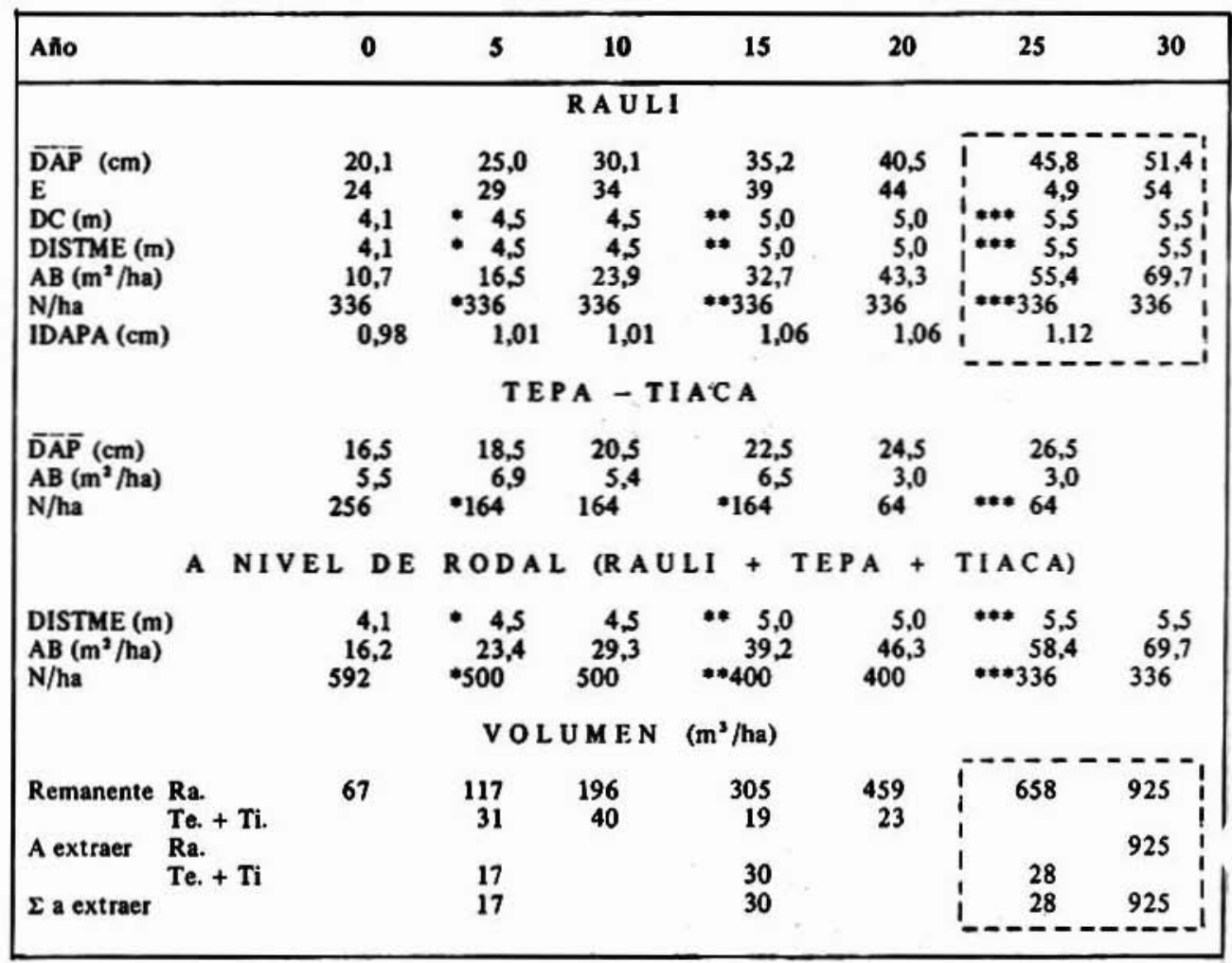

- : A los 5 años salen 92 tepas y tiacas $(\overline{\mathrm{DAP}}=18,5)$.

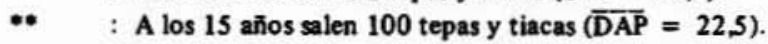

$\bullet$ : A los 25 años salen 64 tepas y tiacas ( $\bar{D} \overline{A P}=26,5)$.

La proyección para tepas y tiacas se proyectó con un crecimiento de $0.4 \mathrm{~cm}$ por año. 


\section{HANS GROSSE W.}

\section{Discusión acerca de las intervenciones a renovales de rauli}

Las dos situaciones (de renoval con predominancia de raulí) analizadas y proyectadas ponen en evidencia la ventaja de un raleo temprano. A los 24 años de edad el incremento diamétrico supera en un $24 \%-28 \%$ al obtenido a los 36 .

Aderıás, la situación de menor edad se vio favorecida por una intervención anterior, que había eliminado a los árboles competidores.

En el rodal más joven, los diámetros alcanzados en un período de 30 años y que bordean los $50 \mathrm{~cm}$, indican la potencialidad de estos bosques para la producción de madera de la mejor calidad.

En el rodal intervenido a mayor edad ( 36 años), aunque su reacción frente al raleo es menor, en 20 años se logra un diámetro medio de aproximadamente $40 \mathrm{~cm}$.

La alternativa de manejo propuesta por situación, es sólo una de muchas opciones de manejo a las que podrían someterse los renovales. Por ejemplo podría resultar atractivo mantener las especies de mayor tolerancia a la falta de luz bajo dosel, para incorporarlas a la generación siguiente.

\section{El crecimiento de renovales de roble}

Los rodales seleccionados para el estudio de la especie Roble, presentan dos situaciones tipo:

- Rodal puro de roble de 41 años de edad con intervención suave anterior

- Rodal de roble mixto de 31 años de edad.

\section{Proyección de un rodal de roble puro (Edad: 41 años)}

El rodal de roble puro presenta 1.040 árboleșpor ha con un área basal de $27 \mathrm{~m}^{2}$.

Considerando las distribuciones en altura, en área basal y en frecuencia, se clasificó el rodal en cuatro sectores. (Figura 5 y cuadro 4). Dentro de éstos, el sector de los individuos dominantes y codominantes, ubicados entre las clases DAP 16 y 28 (sector C), es el que deberá proyectarse a futuro. Los árboles de las clases DAP 44 (sector D) y clase DAP 14 (sector B) se consideran como competidores para el grupo $\mathrm{C}$ y deberán eliminarse.

Los individuos de las clases diamétricas inferiores a $14 \mathrm{~cm}$ se encuentran en posición intermedia y suprimida. Para este ejemplo no se consideran como competencia ni tampoco en la proyección.

La proyección para el rodal remanente (sector $\mathrm{C}$ ) se planteó para un período de 15 años. No se excedió este rango para estar fuera del alcance confiable del modelo de crecimiento utilizado. (Cuadro 5).

A los diez años, mediante un raleo, se obtienen $57 \mathrm{~m}^{3} /$ ha. Finalmente, después de 15 años se pueden explotar $327 \mathrm{~m}^{3}$, constituidos por 450 árboles con un DAP medio de $32 \mathrm{~cm}$.

Suponiendo que el crecimiento diametral continúa con la misma tendencia, de aproximadamente $1 \mathrm{~cm}$ por año, en los años siguientes al período planteado se lograría un considerable incremento del volumen. Si se considera un período de 25 años en los términos de crecimiento planteados, se podría esperar un volumen de cosecha que bordee $105700 \mathrm{~m}^{3} / \mathrm{ha}$. 
FIGURA 5 A, B, C,

ESTADO DEL RENOVAL AL AÑO 0

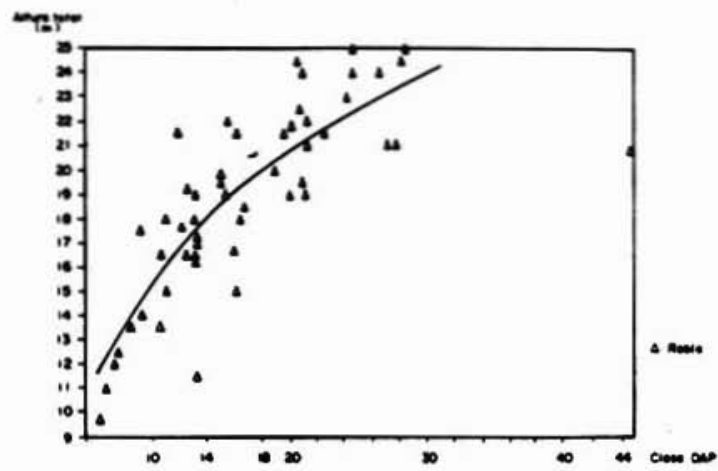

(Especie : Roble)

(Edad : 40 afios)

(Sector : Los Hornos)

A : Altura Total (m) - DAP

B : Area Basal $\left(\mathrm{m}^{2}\right) \cdot$ DAP

C : Tabla de Rodal

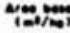
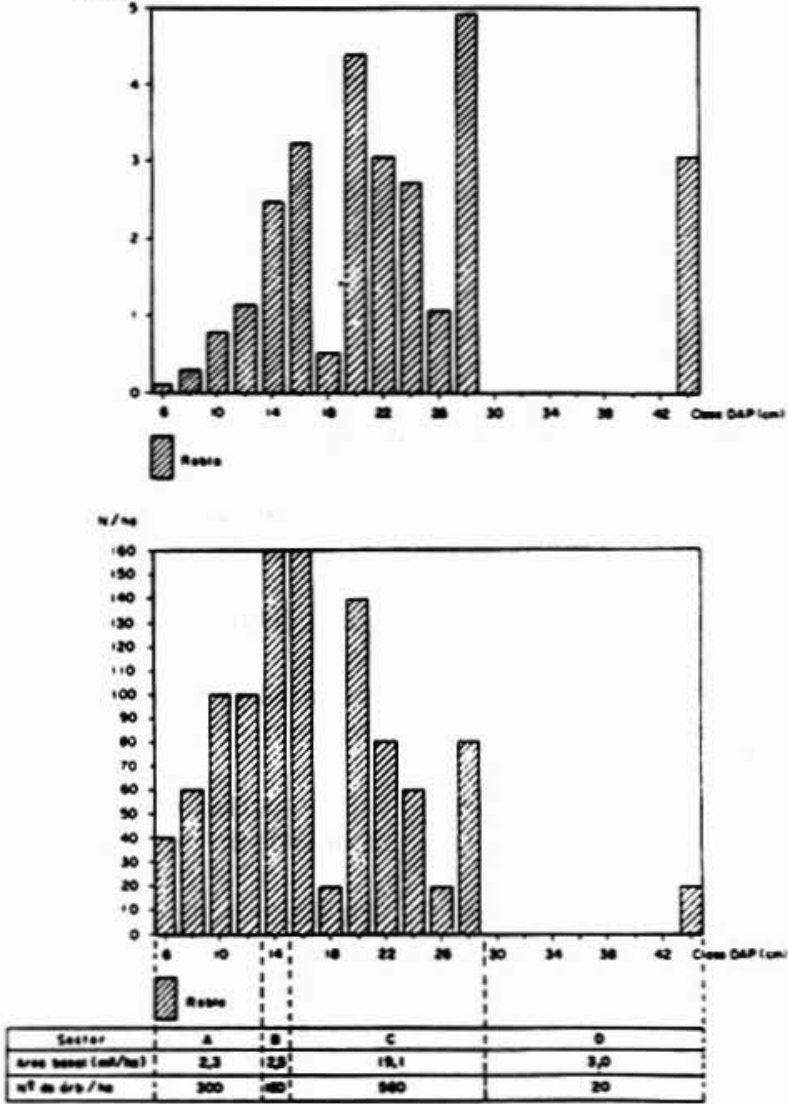
HANS GROSSE W.

\section{CUADRO 4 \\ INTERVENCION AL RENOVAL - AÑO 0 (ROBLE)}

(Edad: 41 Años, Sector Los Hornos)

\begin{tabular}{|l|c|c|c|c|}
\hline \multicolumn{1}{|c|}{$\begin{array}{c}\text { Situac. después de la } \\
\text { intervención }\end{array}$} & Rango & $\begin{array}{c}\text { Area basal } \\
\mathbf{m}^{2} / \mathbf{h a}\end{array}$ & $\begin{array}{c}\% \text { Area basal } \\
\text { relac. a B+C+D }\end{array}$ & N/ha \\
\hline $\begin{array}{l}\text { Estrato en envejecimiento } \\
\text { (a cosechar - anillar) }\end{array}$ & D & 3,0 & 12,2 & 20 \\
Estrato a extraer & B & 2,5 & 10,2 & 160 \\
$\begin{array}{l}\text { Estrato remanente } \\
\text { (A proyectar) }\end{array}$ & C & 19,1 & 77,6 & 560 \\
$\begin{array}{l}\text { Estrato remanente } \\
\text { (Interm. y Suprimido) }\end{array}$ & $\mathrm{A}$ & 2,3 & & 300 \\
Total & $\mathrm{A}+\mathrm{B}+\mathrm{C}+\mathrm{D}$ & 26,9 & & 1.040 \\
\hline
\end{tabular}

\section{CUADRO 5}

PROYECCION ROBLE

Sector Los Hornos

\begin{tabular}{|c|c|c|c|c|}
\hline Año & $\mathbf{0}$ & 5 & 10 & 15 \\
\hline \multicolumn{5}{|c|}{ ROBLE (RODAL) } \\
\hline$\overline{\mathrm{DAP}}(\mathrm{cm})$ & 20,9 & 23,8 & 27,2 & 31,8 \\
\hline E & 41 & 46 & 51 & 56 \\
\hline DISTME (m) & 4,2 & 4,2 & 4,7 & 4,7 \\
\hline $\mathrm{N} / \mathrm{ha}$ & 560 & 560 & 450 & 450 \\
\hline $\mathrm{AB}\left(\mathrm{m}^{2} / \mathrm{ha}\right)$ & 19,2 & 24,9 & 26,2 & 35,7 \\
\hline \multirow[t]{2}{*}{ IDAPA $(\mathrm{cm})$} & 0,57 & 0,67 & 0,91 & 1,21 \\
\hline & \multicolumn{2}{|c|}{ VOLUMEN $\left(\mathrm{m}^{3} / \mathrm{ha}\right)$} & \multicolumn{2}{|c|}{ ' } \\
\hline Remanente & 164 & 219 & 234 & 327 \\
\hline A extraer & * 48 & & 57 & 327 \\
\hline
\end{tabular}

: Volumen a extraer del sector B y D, correspondiente a las clases diamétricas 14 y 44.
i... Valores extrapolados. 
Proyección de un rodal de roble-rauli (Edad: 31 años)

El rodal de raulí-roble está constituido por 944 árboles/ha de los cuales el $75 \%$ correspon. den en igual proporción a las especies Roble y Raulí. Los individuos restantes son coigües, tepas, tineos $\mathrm{y}$ avellanos.

Para la proyección del rodal, este se dividió en estrato suprimido - intermedio hasta la clase DAP 10 (A), estrato codominante y dominante a proyectar entre las clases DAP 12 a 28 (B) y estrato dominante a eliminar sobre la clase DAP 30 (C). (Cuadro 6 y Figura 6).

El estrato a eliminar está constituido por árboles de gran tamaño en relación a los árboles a proyectar. La escasa luz disponible para estos últimos se refleja en crecimientos diamétricos bajo $0,5 \mathrm{~cm} /$ año durante sus primeros 31 años. El grupo de árboles remanentes a proyectar está constituido por 592 individuos, de los cuales hay 192 robles, 192 raulíes y 208 árboles entre coigües, tepas, tineos y avellanos.

La proyección se planteó para un período de 20 años. No se excedió este lapso por limita. ciones en el modelo de crecimiento.

Al inicio del período, el volumen a extraer es de $247 \mathrm{~m}^{3}$, correspondiente a coigües, tineos y avellanos. Después de 10 años se extraen $59 \mathrm{~m}^{3}$ de roble - raulí. Finalmente, al término del período (20 ańos), se explotan $150 \mathrm{~m}^{3}$ de roble y $78 \mathrm{~m}^{3}$ de raulí.

Es de suponer que en un período de 30 años, considerando la realización de raleos, los árboles alcanzarían diámetros medios de al menos $40-50 \mathrm{~cm}$.

\section{CUADRO 6 \\ INTERVENCION AL RENOVAL - AÑO 0 (ROBLE) \\ (Edad: 31 Años, Sector FUY)}

\begin{tabular}{|l|c|c|c|c|}
\hline \multicolumn{1}{|c|}{$\begin{array}{c}\text { Situac. después de la } \\
\text { intervención }\end{array}$} & Rango & $\begin{array}{c}\text { Area basal } \\
\mathrm{m}^{2} / \mathbf{h a}\end{array}$ & $\begin{array}{c}\text { \% Area basal en } \\
\text { relac. a B+C }\end{array}$ & N/ha \\
\hline Estrato suprim. - interm. & A & 1,7 & & 288 \\
Estrato a proyectar & B & 12,6 & 32,2 & 592 \\
$\begin{array}{l}\text { Estrato a cosechar } \\
\text { (anillar) en envejecim. }\end{array}$ & C & 26,4 & 67,7 & 64 \\
Total & A+B+C & 40,7 & & 944 \\
\hline
\end{tabular}


HANS GROSSE W.

FIGURA 6

\section{A, B, C: ESTADO DEL RENOVAL AL AÑO 0}

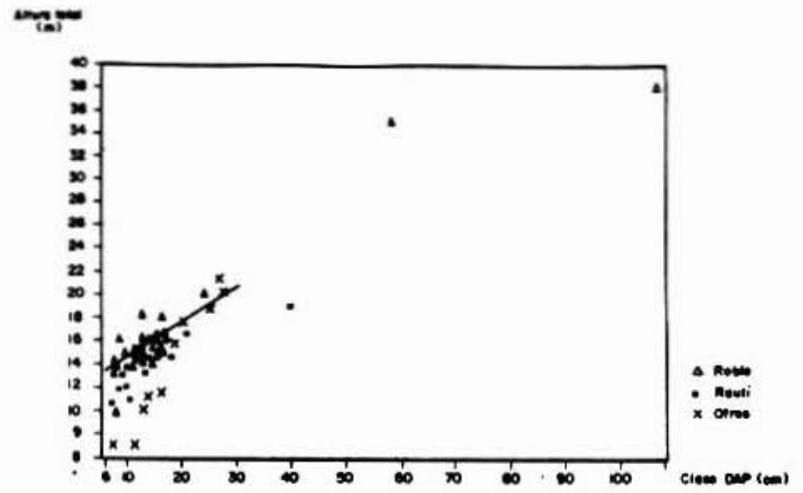

$\begin{array}{lll}\text { (Especie } & : \text { Roble) } \\ \text { (Edad } & : \text { 31 Años) } \\ \text { (Sector } & : \text { Pto. Fuy) }\end{array}$

A : Altura Total $(m) \quad-$ DAP
B : Area Basal $\left(m^{2}\right)$
C : TABLA DE RODAL

4

is

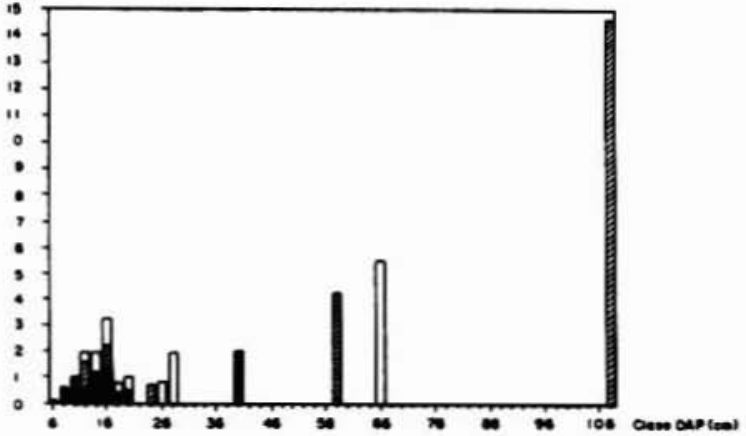

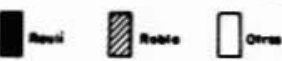

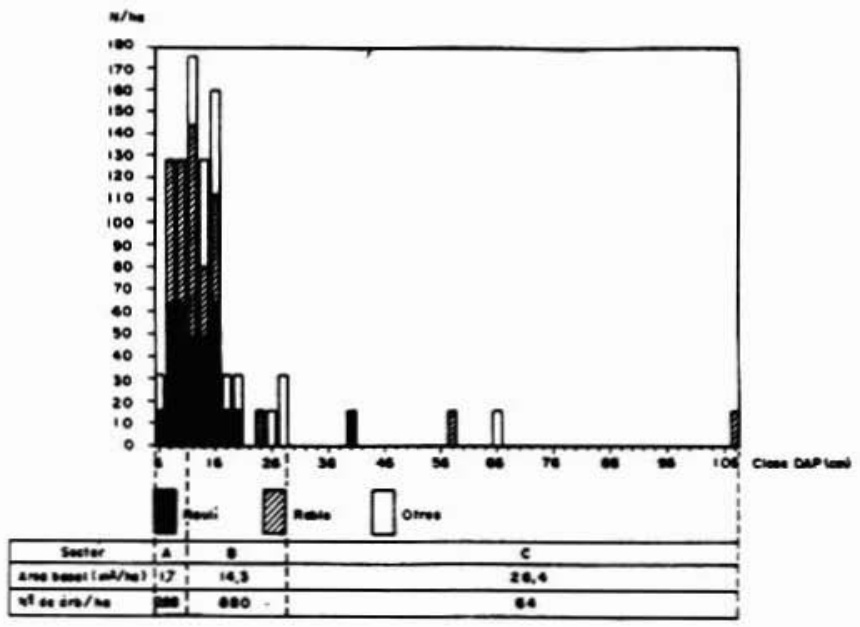




\section{CUADRO 7}

\section{PROYECCION ROBLE}

Sector Fuy

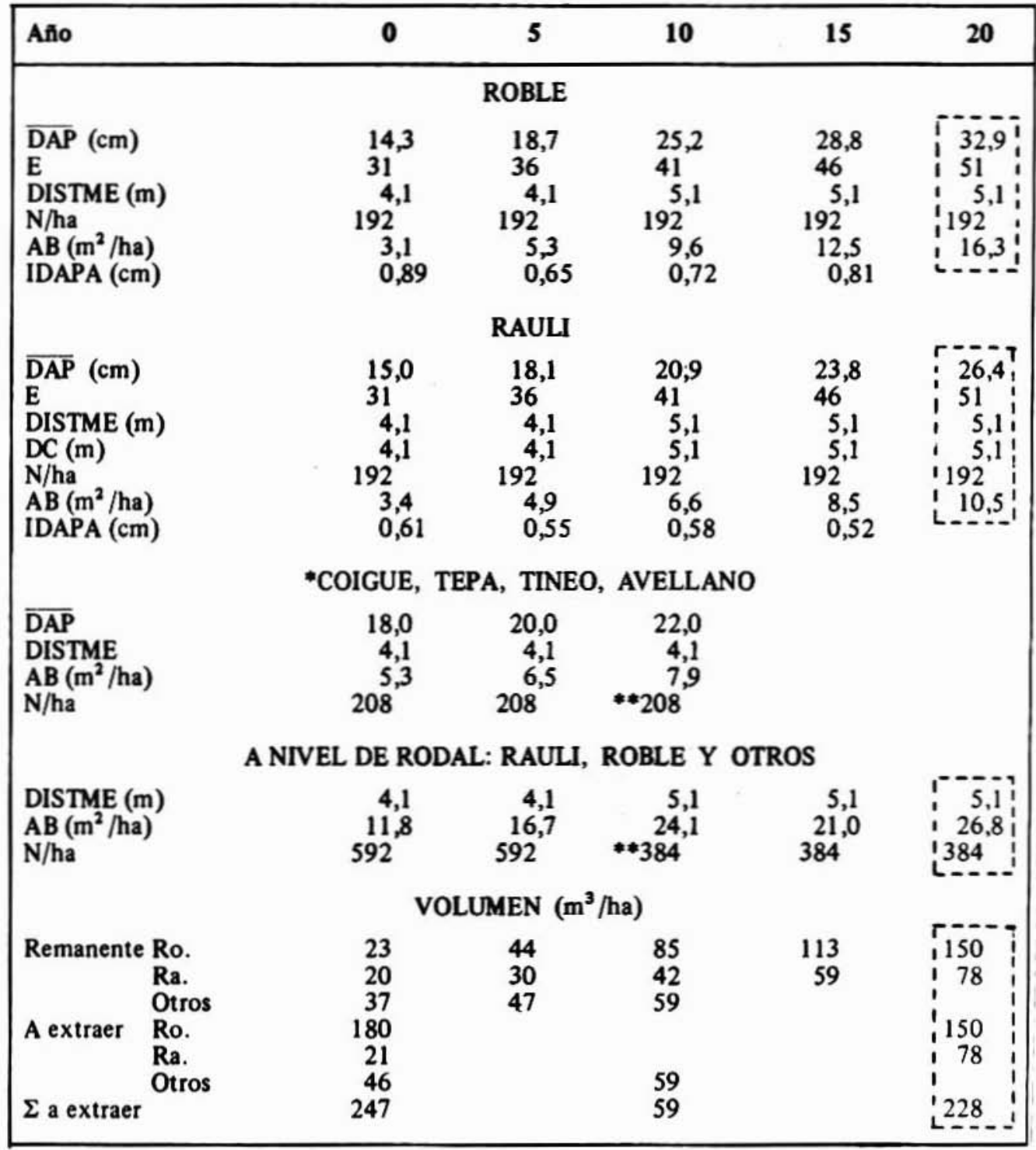

- : La proyección de coigüe, tepa, tineo y avellano se hizo considerando $0.4 \mathrm{~cm}$ de incremento dia. metral anual.

*. : Se extraen 208 árboles de Co., Te., Ti. y Av. con un DAP = 22,0 cm.

i... : Valores extrapolados. 


\section{Discusión acerca de las intervenciones a renovales de roble}

Los dos rodales estudiados, a pesar de contar con un raleo suave, realizado diez años antes de la medición, presentan un número de árboles demasiado alto como para aumentar en forma espontánea la velocidad de crecimiento de los individuos. En ambos casos los árboles a proyectar tuvieron un crecimiento en diámetro de $0,5 \mathrm{~cm}$ por año, en períodos de 31 y 41 años, respectivamente. El hecho de liberarlos de sus competidores más directos permite incrementar el crecimiento diametral en un $50 \%$.

El modelo de crecimiento diametral utilizado tiene validez sólo hasta los 46 años de edad, por consiguiente la proyección se realizó para un período de 15 y 20 años para los rodales de Los Hornos y Fuy respectivamente.

Sin embargo, en base a la gran capacidad de reacción de la especie frente a un mayor espaciamiento, aún a edad avanzada, podemos suponer que el incremento diametral se mantendría entre $0,8-1 \mathrm{~cm} /$ año. Por consiguiente si se alarga el período a 30 años, se lograrían diámetros medios de aproximadamente $40 \mathrm{~cm}$.

El futuro para ambas situaciones debería definirse mediante el manejo de una combinación del material rebrotado y regeneración artificial o plantación.

\section{El crecimiento de renovales de coigüe}

Los rodales de coigüe elegidos para el estudio corresponden a dos situaciones tipo:

- Rodal de coigüe, con avellanos y tiacas en el estrato inferior (Edad: 40 años).

- Rodal de coigüe, con tepas bajo dosel (Edad: 46 años)

Proyección de un rodal de coigüe - avellano - tiaca (Edad: 40 años)

El rodal de coigüe - avellano - tiaca presenta 3.400 árboles por ha, de los cuales $48 \%$ son coigües. No hubo intervención previa. El área basal es de $47 \mathrm{~m}^{2} /$ ha, y orienta sobre la ocupación del sitio para coigüe a la edad de 40 años.

El rodal se subdividio según el estado de desarrollo de los árboles en cuatro sectores.

El sector A agrupa a los árboles de los doseles inferiores, el sector $\mathrm{B}$ comprende los árboles dominantes y codominantes a extraer dentro de las clases diamétricas $16-18$. El sector a proyectar (C) está formado por árboles dominantes y codominantes (clases 20 - 30). Los árboles de las clases diamétricas 34 - 36 conforman el sector D y corresponden a árboles dominantes (árboles lobo) que serán extraídos (Cuadro 8 y Figura 7).

El rodal se proyectó a un período de 15 años. No se excedió este rango por las limitantes de estimación que presenta el modelo de crecimiento diametral sobre la edad de 50 años.

Se comienza con una extracción de $96 \mathrm{~m}^{3} / \mathrm{ha}$, principalmente de coigüe. Al finalizar un período de 15 años, se podrían explotar aproximadamente $250 \mathrm{~m}^{3} /$ ha de esta especie con diámetros medios de $30 \mathrm{~cm}$.

A pesar de que se liberó el estrato a proyectar de sus competidores más directos, el incremento diamétrico sólo alcanza $0,4 \mathrm{~cm}$ (Cuadro 9).

$\mathrm{Si}$ se proyecta el rodal por un período de 30 años, suponiendo un incremento diamétrico de $0,3 \mathrm{~cm}$ anuales, el diámetro medio podría superar los $35 \mathrm{~cm}$. El volumen a esperar sería de aproximadamente $340 \mathrm{~m}^{3} / \mathrm{ha}$. 
CUADRO 8

INTERVENCION AL RENOVAL - AÑO 0 (COIGUE)

(Edad: 40 Años, Sector Truful)

\begin{tabular}{|l|c|c|c|c|}
\hline \multicolumn{1}{|c|}{$\begin{array}{c}\text { Situac. después de la } \\
\text { intervención (Area basal) }\end{array}$} & Rango & $\begin{array}{c}\text { Area basal } \\
\mathbf{m}^{2} / \mathbf{h a}\end{array}$ & $\begin{array}{c}\% \text { Area basal en } \\
\text { relac. a B+C+D }\end{array}$ & N/ha \\
\hline Estrato Domin, y Codom. & B+C+ D & 30,4 & 100,0 & 760 \\
Estrato a extraer & B+ D & 12,4 & 40,8 & 700 \\
Estratoremanente (DomyCod.) & C & 18,0 & 59,2 & 400 \\
Estrato remanente (Interm. & A & 17,1 & & 2.640 \\
y Suprim) & & & & 3.400 \\
Total & A+ B+C+D & 47,5 & & \\
\hline
\end{tabular}

CUADRO 9

PROYECCION COIGUE

Sector Truful)

\begin{tabular}{|c|c|c|c|c|}
\hline Afio & $\mathbf{0}$ & 5 & 10 & 15 \\
\hline \multicolumn{5}{|c|}{ COIGUE } \\
\hline$\overline{\mathrm{DAP}}(\mathrm{cm})$ & 23,8 & 26,2 & 28,3 & 30,2 \\
\hline $\mathbf{E}$ & 40 & 45 & 50 & 55 \\
\hline Hิ. TOT. (m) & 18,6 & 19,7 & 20,2 & 20,6 \\
\hline DISTME (m) & 5 & 5 & 5 & 5 \\
\hline $\mathrm{AB}\left(\mathrm{m}^{2}\right)$ & 17,8 & 21,6 & 25,2 & 28,7 \\
\hline N/ha & 400 & 400 & 400 & 400 \\
\hline IDAPA (cm) & 0,47 & 0,42 & 0,38 & \\
\hline \multicolumn{5}{|c|}{ VOLUMEN $\left(\mathrm{m}^{3} / \mathrm{ha}\right)$} \\
\hline Remanente & 145 & 180 & 214 & 247 \\
\hline A extraer Co. & 93 & & & 247 \\
\hline A extraer Otros & 3 & & & \\
\hline
\end{tabular}

: Valores extrapolados.
: Valores estimados. 
FIGURA 7 A, B,C,

\section{ESTADO DEL RENOVAL AL AÑO 0}

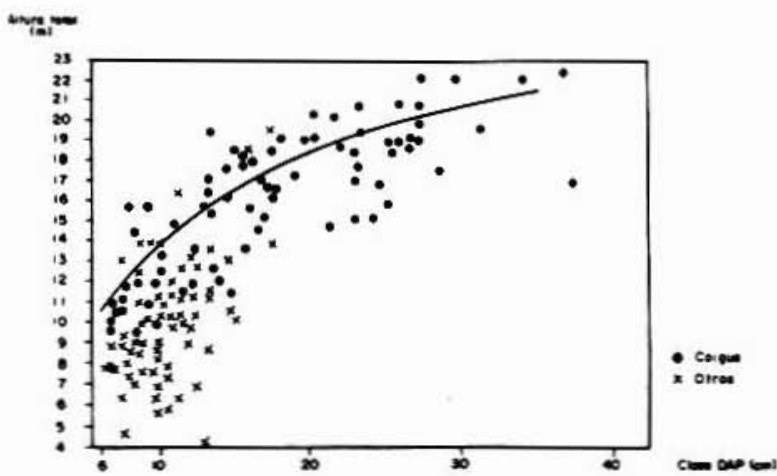

(Especie : Coigue)

(Edad : 40 años)

(Sector : Truful)

A : Altura Total (m) - DAP

B : Area Basal $\left(\mathrm{m}^{2}\right) \cdot$ DAP

C : Tabla de Rodal
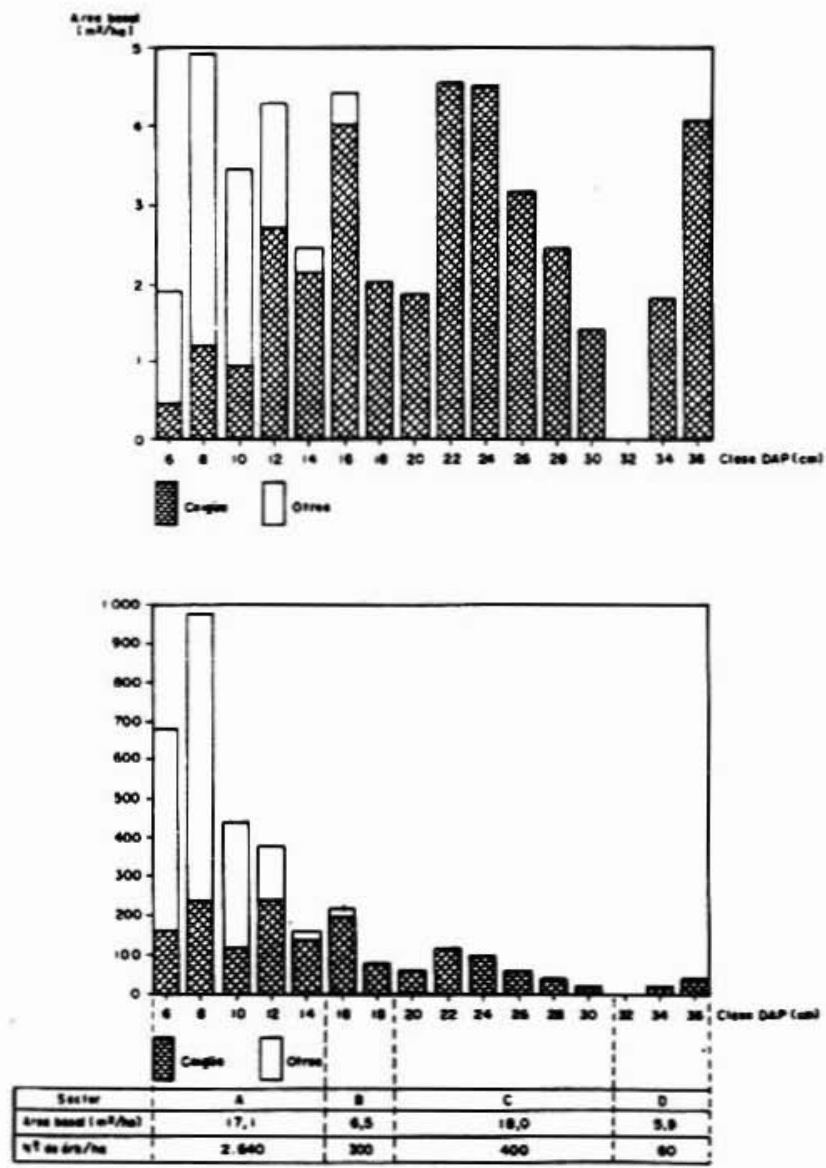


\section{Proyección de un rodal de coigüe - tepa (Edad: 46 años)}

El rodal de coigüe - tepa presenta 760 árboles/ha con una área basal de $57 \mathrm{~m}^{2} / \mathrm{ha}$. De éstos el $58 \%$ son coigües, el $32 \%$ tepas y el resto robles y raulíes. No se realizó raleo previo a la medición. El rodal se subsidivió según el estado de desarrollo de los árboles. Entre las clases DAP 6 18 (sector A), se encuentra una fuerte presencia de tepas, con 220 individuos.

Los sectores B (CI. DAP 20 - 24) y D (CI. DAP 48 - 60) comprenden a los individuos que deberán eliminarse. Los árboles a proyectar se encuentran ubicados entre la clase DAP 28 y la 48 (sector C) (Cuadro 10 y Figura 8).

Los incrementos diamétricos a obtener con el esquema propuesto, se acercan a $1 \mathrm{~cm}$. Por consiguiente, al cabo de 10 años el diámetro medio sería de aproximadamente $50 \mathrm{~cm}$ para los 240 coigües remanentes (Cuadro 11).

En términos de volumen, al inicio del período se extraen 120 y $12 \mathrm{~m}^{3} /$ ha de Coigüe y Roble respectivamente. Después de 10 años, es decir, finalizando el período de proyección, se pueden explotar $420 \mathrm{~m}^{3} /$ ha de Coigüe y $13 \mathrm{~m}^{3}$ /ha de Tepa.

En el dosel suprimido - intermedio las tepas deben ser consideradas como un componente valioso de la generación siguiente. Podrían ser mezcladas en el futuro con plantaciones de árboles del género Nothofagus.

CUADRO 10

INTERVENCION AL RENOVAL - AÑO 0 (COIGUE)

(Edad: 46 Años, Sector Los Hornos)

\begin{tabular}{|l|c|c|c|c|}
\hline \multicolumn{1}{|c|}{$\begin{array}{c}\text { Situac. después de la } \\
\text { intervención (Area basal) }\end{array}$} & Rango & $\begin{array}{c}\text { Area basal } \\
\mathbf{m}^{2} / \mathbf{h a}\end{array}$ & $\begin{array}{c}\% \text { Area basal en } \\
\text { relac. a B+C+D }\end{array}$ & N/ha \\
\hline Estratos Domin. y Codom. & B+C+ D & 52,6 & 100,0 & 440 \\
Estrato a extraer & B+ D & 19,8 & 38,0 & 180 \\
Estratoremanente (Dom.yCod.) & C & 32,8 & 62,0 & 260 \\
$\begin{array}{l}\text { Estrato remanente (Interm. } \\
\text { y Suprim.) }\end{array}$ & A & 4,1 & & 320 \\
Total & A+ B+C+D & $56,7$. & & 760 \\
\hline
\end{tabular}


FIGURA 8 A, B,C

ESTADO DEL RENOVAL AL AÑO 0

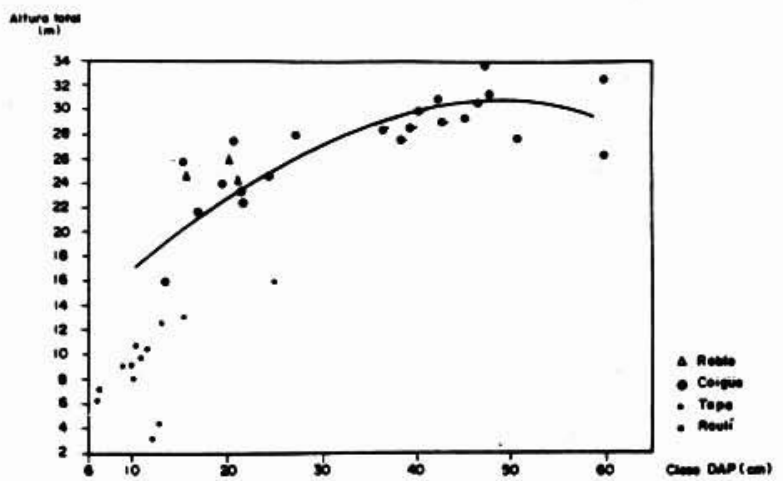

(Especie : Coigue)

(Edad : 46 años)

(Sector : Los Hornos)

A : Altura Total (m) - DAP

B : Area Basal $\left(\mathrm{m}^{2}\right)$ - DAP

C : Tabla de Rodal

(andina)
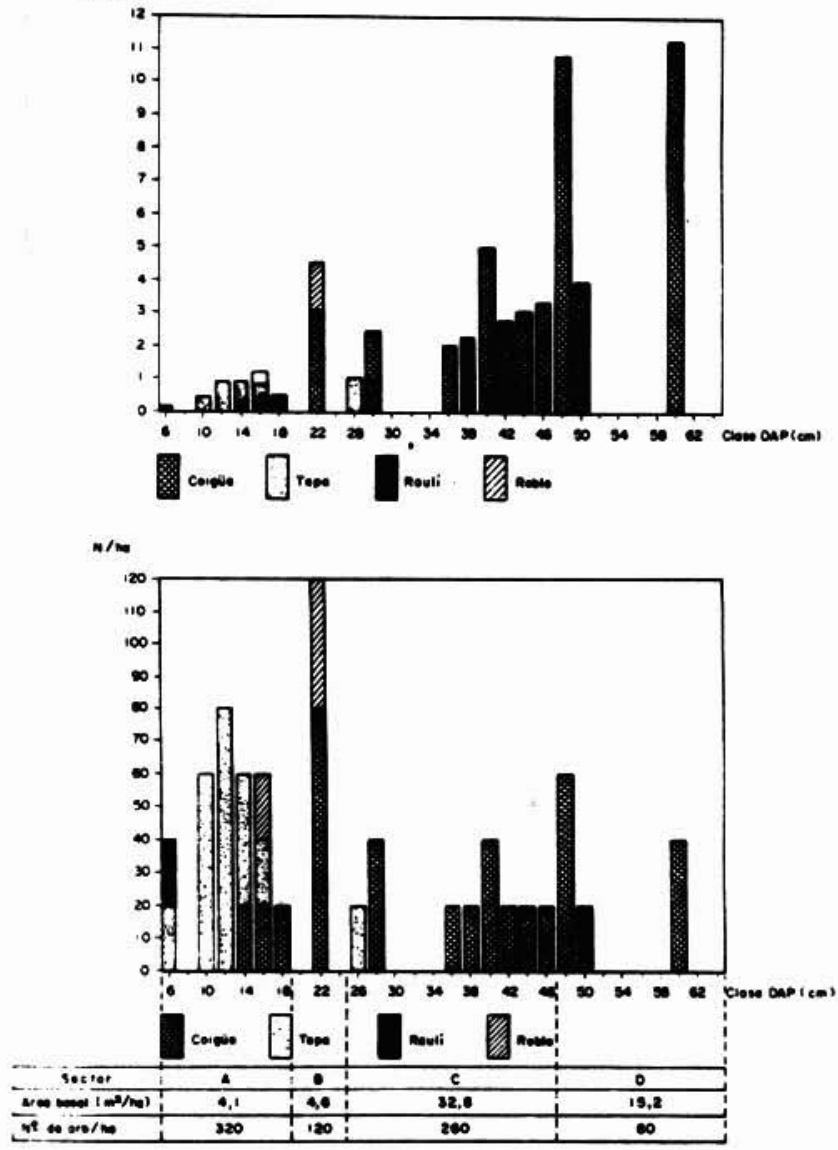


\section{CUADRO 11 \\ PROYECCION COIGUE \\ Sector Los Hornos}

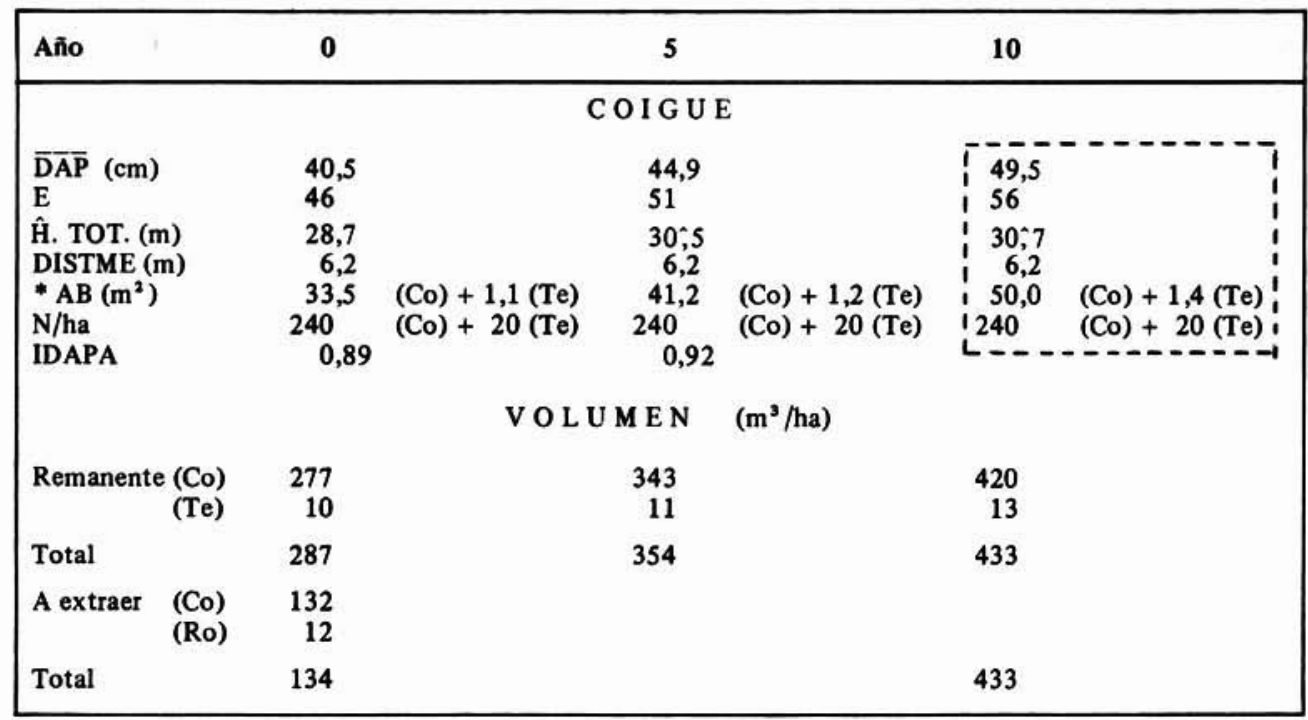

* : La tepa se proyecta con un incremento de $0,4 \mathrm{~cm}$ al año.

I. I : Valores extrapolados.

: Valores estimados 


\section{Discusión acerca de las intervenciones a renovales de coigüe}

Las dos situaciones estudiadas presentan condiciones de sitio diferentes. Esto queda de manifiesto al observar las alturas medias alcanzadas por los coigües, que en el caso de Truful es de $18,6 \mathrm{~m}$ y en Los Hornos $28,7 \mathrm{~m}$, considerando una edad similar en ambos rodales.

Por otro lado, los incrementos diamétricos promedio fueron de $0,4 \mathrm{~cm} /$ año para el rodal de Truful y de $0,9 \mathrm{~cm} / \mathrm{año}$ para Los Hornos.

Diferencias de sitio tan marcadas, implican objetivos de manejo distintos. En el sitio de rendimiento más alto (Los Hornos) el objetivo deberá apuntar a producir árboles de grandes diámetros, para obtener madera de calidad. En el sitio de menor rendimiento deberá optarse por maximizar el volumen, sin intervenciones intermedias de alto costo.

El futuro de las especies acompañantes en el estrato inferior, dependerá de su valor comercial y rendimiento. Si estas son avellanos y tiacas, en el ejercicio se optó por su eliminación para incentivar el crecimiento de la especie principal, coigüe. Si las especies acompañantes en el estrato bajo son tepas, que por su forma y dimensión podrían ser comercialmente atractivas, es recomendable mantenerlas. Una vez que se explotó el coigüe, es factible manejar el rodal con una mezcla de especies, dentro de las cuales las tepas tomarían un rol preponderante. En los sectores abiertos se pueden incorporar, según los objetivos de la empresa, especies del género Nothofagus.

\section{El crecimiento de renovales de tepa}

Los rodales de tepa elegidos corresponden a las siguientes dos situaciones tipo:

- Rodal de tepa de 63 años, dominado por un estrato superior de roble. Ambos estratos están en la misma situación de ocupación de sitio.

- Rodal de tepa de 81 años, se encuentra dominado por un estrato superior de coigüe de gran participación en Area Basal.

\section{Proyección de un rodal de tepa bajo dosel de roble (Edad: 63 años)}

El rodal de tepa bajo escaso dosel de roble presenta 3.500 individuos por ha con un área basal de $79 \mathrm{~m}^{2} / \mathrm{ha}$. El $90 \%$ de los árboles son tepas, mientras que el resto se divide en robles y otras especies. En términos de área basal la participación de tepa es del $80 \%$.

La mayoría de las tepas tienen un DAP inferior a $34 \mathrm{~cm}$, a excepción de algunos individuos que se encuentran en la clase diamétrica 100. Estos, junto a árboles de otras especies conforman el estrato dominante, el que se extraerá al inicio del período (Cuadro 12, Sector D).

El sector a proyectar (C) está comprendido entre las clases diamétricas $18 \cdot 34$, agrupando tepas y otras especies. Estas últimas también serán eliminadas al inicio del período.

Las tepas de las clases DAP 12 a 18 son competidores importantes para el estrato remanente (C), razón por la cual también se eliminan al inicio. Los individuos ubicados bajo la clase DAP $12 \mathrm{~cm}$, son árboles intermedios y suprimidos que no se consideran como competidores de importancia en esta etapa.

La eliminación del estrato dominante en envejecimiento con $31 \mathrm{~m}^{2}$ de área basal, puede lograrse a través de la extracción y de anillamiento de los árboles. otros).

$\mathrm{Al}$ año de inicio del período, se pueden extraer $275 \mathrm{~m}^{3} / \mathrm{ha}$ (49\% tepa, $42 \%$ roble, $9 \%$

La proyección de las tepas, en un período de 30 años permite obtener un DAP medio de $31 \mathrm{~cm}$ y una extracción final de $356 \mathrm{~m}^{3} / \mathrm{ha}$. 
CUADRO 12

INTERVENCION AL RENOVAL - AÑO 0 (TEPA)

(Edad: 63 Años, Sector Moloo)

\begin{tabular}{|l|c|c|c|c|}
\hline \multicolumn{1}{|c|}{$\begin{array}{c}\text { Situac. después de la } \\
\text { intervención (Area basal) }\end{array}$} & Rango & $\begin{array}{c}\text { Area basal } \\
\mathbf{m}^{2} / \mathbf{h a}\end{array}$ & $\begin{array}{c}\text { \% Area basal en } \\
\text { relac. a B+C+C' }\end{array}$ & N/ha \\
\hline $\begin{array}{l}\text { Estrato dominante y codom. } \\
\text { en envejecimiento } \\
\text { A extraer }\end{array}$ & D & 30,9 & & 160 \\
$\begin{array}{l}\text { Estrado remanente dominante } \\
\text { y codominante a proyectar }\end{array}$ & C & 24,8 & 72,7 & 600 \\
$\begin{array}{l}\text { Indiv. a extraer del estrato C } \\
\begin{array}{l}\text { Estrato codominante- } \\
\text { intermedio } \\
\text { A extraer }\end{array}\end{array} \quad$ C' & 2,9 & 7,6 & 60 \\
$\begin{array}{l}\text { Estrato intermedio- } \\
\text { suprimido } \\
\text { Remanente }\end{array}$ & $\mathrm{B}$ & 10,4 & 27,3 & 720 \\
\begin{tabular}{l} 
Total \\
\hline
\end{tabular} & $\mathrm{A}+\mathrm{B}+\mathrm{C}+\mathrm{C}^{\prime}+\mathrm{D}$ & 78,7 & & 3.280 \\
\hline
\end{tabular}


FIGURA 9 A, B,C

\section{ESTADO DEL RENOVAL AL AÑO 0}

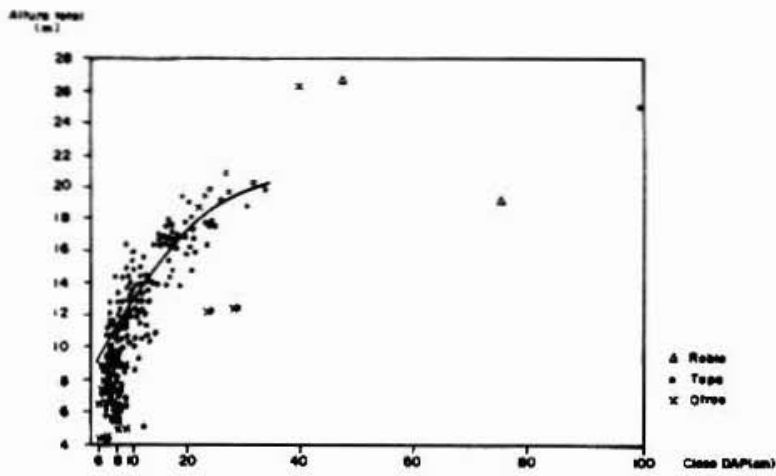

(Especie : Tepa)

(Edad : 63 años)

(Sector : Molco)

A : Altura Total (m) - DAP

B : Area Basal $\left(\mathrm{m}^{2}\right)$ - DAP

C : Tabla de Rodal

ind

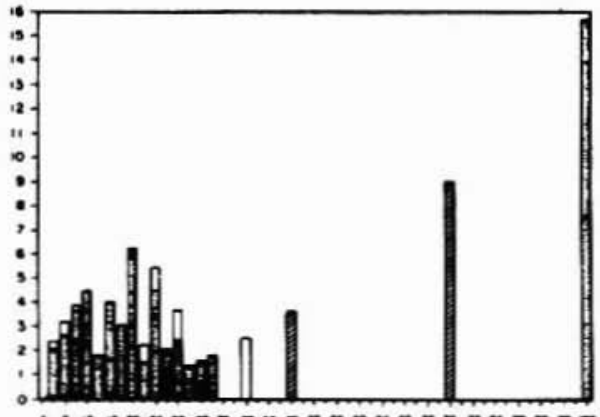

P +

Drom Doum

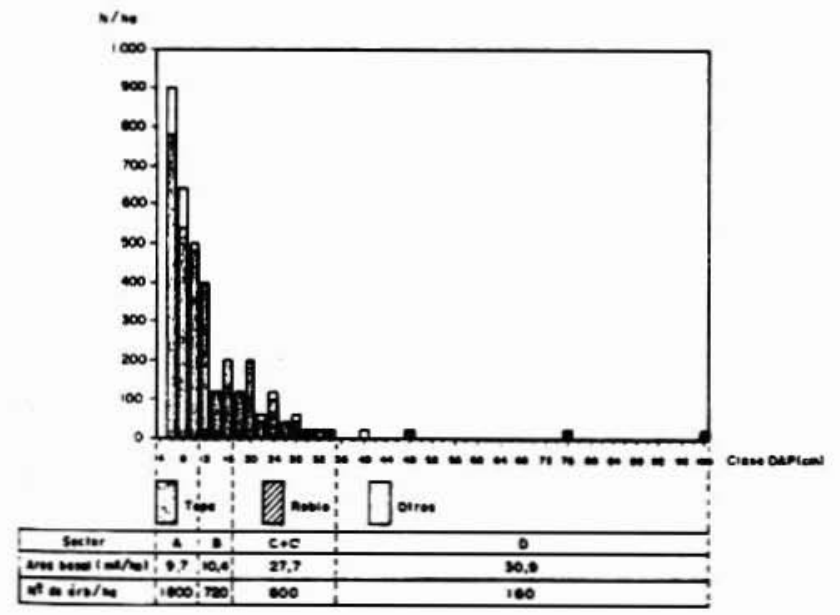


CUADRO 13

PROYECCION TEPA

Sector Molco

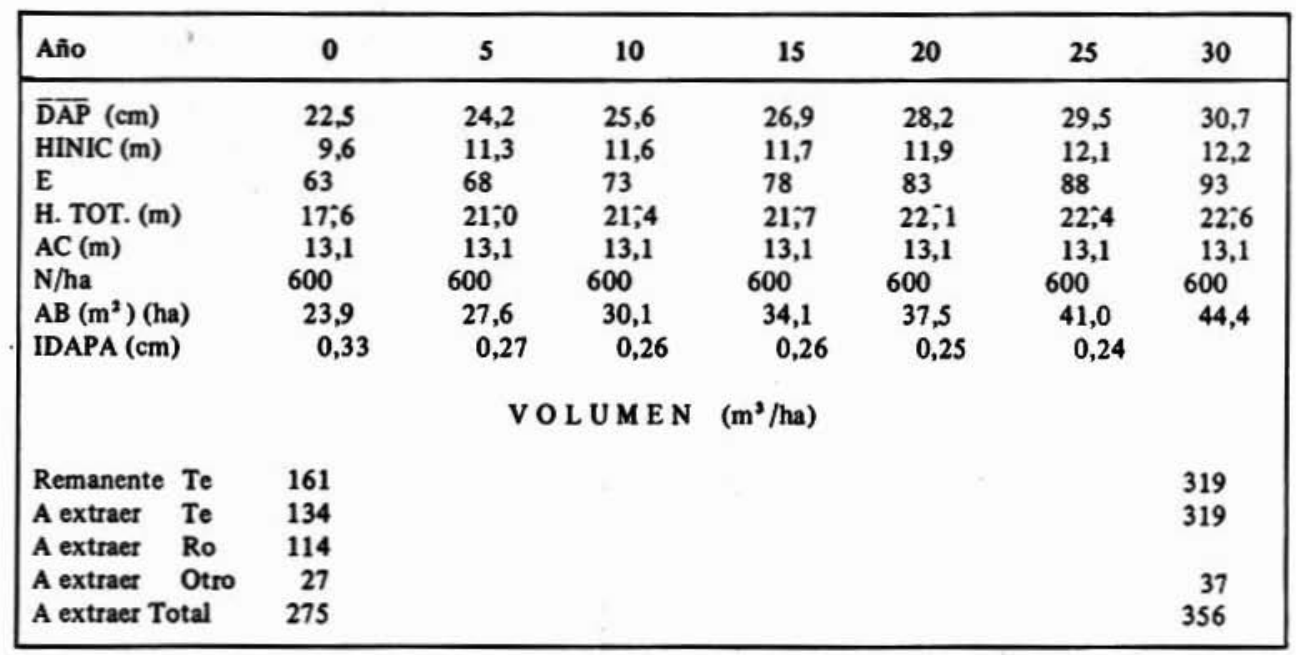

HINIC : Se estimó como 0,54 *H. TOT. La relación se basó en la situación inicial.

: Valores estimados

Proyección de un rodal de tepa fuertemente dominado (Edad: 81 años)

El rodal de tepa bajo dosel de coigüe contiene 1.160 árboles/ha con un área basal de $112 \mathrm{~m}^{2} / \mathrm{ha}$. La dominancia del rodal está dada por 200 coigües con el $84 \%$ del área basal (figura 10). Este estrato (C), se elimina y/o anilla al inicio del periodo para liberar las tepas en el estrato inferior (Cuadro 14).

El estrato a proyectar se localiza entre las clases DAP 10 y $30 \mathrm{~cm}$. Contiene 580 individuos, de los cuales sólo se dejan 480 tepas en pie. Los individuos de la clase DAP 8 o menores se consideran como competidores de poca importancia dejándose en pie. Con el esquema de intervención propuesto el $\mathrm{AB}$ remanente es de $12 \mathrm{~m}^{2}$. La relación entre este valor y el área basal del rodal al inicio del período indica una intervención extremadamente fuerte. Esta podría afectar la estabilidad del rodal a proyectar, sólo si las tepas que lo constituyen presentaran una relación altura - diámetro muy alta. Como esto no ocurre, ya que esta relación es de aproximadamente 0,7 , se puede esperar que los daffos por efectos del viento sean mínimos.

El volumen a extraer al inicio del período es de $844 \mathrm{~m}^{3} /$ ha $(99 \%$ coigüe) y al final del período es de $232 \mathrm{~m}^{3}$ /ha de tepa. $28 \mathrm{~cm}$.

Después de un período de 30 años, las Tepas proyectadas alcanzan un DAP medio de 
HANS GROSSE W.

CUADRO 14

INTERVENCION AL RENOVAL - AÑO O (TEPA)

(Edad: 81 Affos, Sector Los Homos)

\begin{tabular}{|l|c|c|c|c|}
\hline \multicolumn{1}{|c|}{$\begin{array}{c}\text { Situac. después de la } \\
\text { intervencion (Area basal) }\end{array}$} & Rango & $\begin{array}{c}\text { Area basal } \\
\mathbf{m}^{2} / \mathbf{h a}\end{array}$ & $\begin{array}{c}\text { \% Area basal en } \\
\text { relac. al total }\end{array}$ & N/ha \\
\hline $\begin{array}{l}\text { Estrato dominante y codom. } \\
\text { (a extraer - anillar) }\end{array}$ & C & 94,5 & 84,1 & 200 \\
Estrato a proyectar & B & 13,8 & 12,3 & 480 \\
Indiv. a extrar estrato B & B' & 2,5 & 2,2 & 100 \\
Estrato suprimido-intermedio & A & 1,6 & 1,4 & 380 \\
Total & A+ B+ B'+C & 112,4 & 100,0 & 1.160 \\
\hline
\end{tabular}

CUADRO 15

PROYEOCION TEPA

Sector Huilo

\begin{tabular}{|c|c|c|c|c|c|c|c|}
\hline Aflo & 0 & 5 & 10 & 15 & 20 & 25 & 30 \\
\hline $\begin{array}{l}\overline{\text { DAP }}(\mathrm{cm}) \\
\text { HINIC } \\
\text { E } \\
\text { H. TOT. } \\
\text { AC } \\
\text { N } \\
\text { AB } \\
\text { IDAPA }\end{array}$ & $\begin{array}{c}18,2 \\
5,2 \\
81 \\
13 \\
16,4 \\
480 \\
12,5 \\
0,29\end{array}$ & $\begin{array}{c}19,7 \\
5,4 \\
86 \\
14,2 \\
16,4 \\
480 \\
14,6 \\
0,31 \\
\text { v }\end{array}$ & $\begin{array}{c}21,3 \\
5,7 \\
91 \\
15,0 \\
16,4 \\
480 \\
17,1 \\
0,32 \\
\text { L U M E N }\end{array}$ & $\begin{array}{c}22,9 \\
6,0 \\
96 \\
15 ; 8 \\
16,4 \\
480 \\
19,8 \\
0,33 \\
\mathrm{~m}^{3} / \mathrm{ha}\end{array}$ & $\begin{array}{c}24,5 \\
6,3 \\
101 \\
16 ; 6 \\
16,4 \\
480 \\
22,6 \\
0,33\end{array}$ & $\begin{array}{c}26,2 \\
6,6 \\
106 \\
17 ; 5 \\
16,4 \\
480 \\
25,9 \\
0,33\end{array}$ & $\begin{array}{c}27,9 \\
7,0 \\
111 \\
18,3 \\
16,4 \\
480 \\
29,3\end{array}$ \\
\hline
\end{tabular}

HINIC : Se estimó como $0,38{ }^{\star} \mathrm{H}$. Tot. La relación se basó en la situación inicial.

: Valores estimados

Volumen 3, Número 6, 1989/ 65 
FIGURA $10 \mathrm{~A}, \mathrm{~B}, \mathrm{C}$

\section{ESTADO DEL RENOVAL AL AÑO 0}

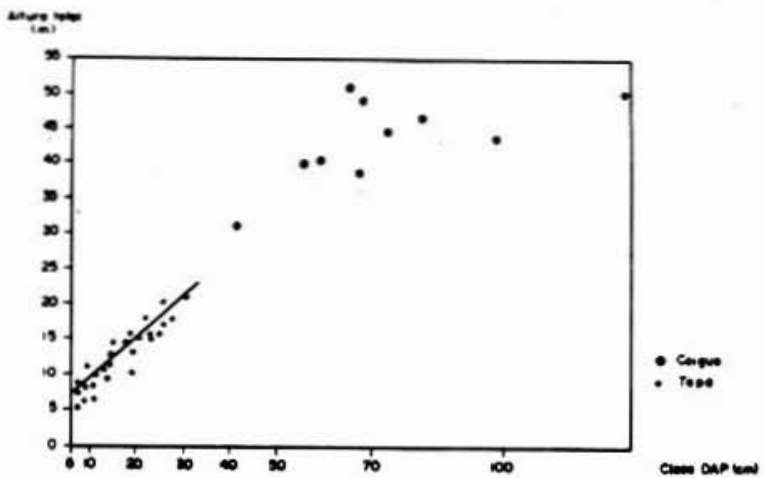

(Especie : Tepa)

(Edad : 81 años)

(Sector : Huilo)
A : Altura Total (m) - DAP
B : Area Basal $\left(\mathrm{m}^{2}\right) \cdot$ DAP
C : Tabla de Rodal
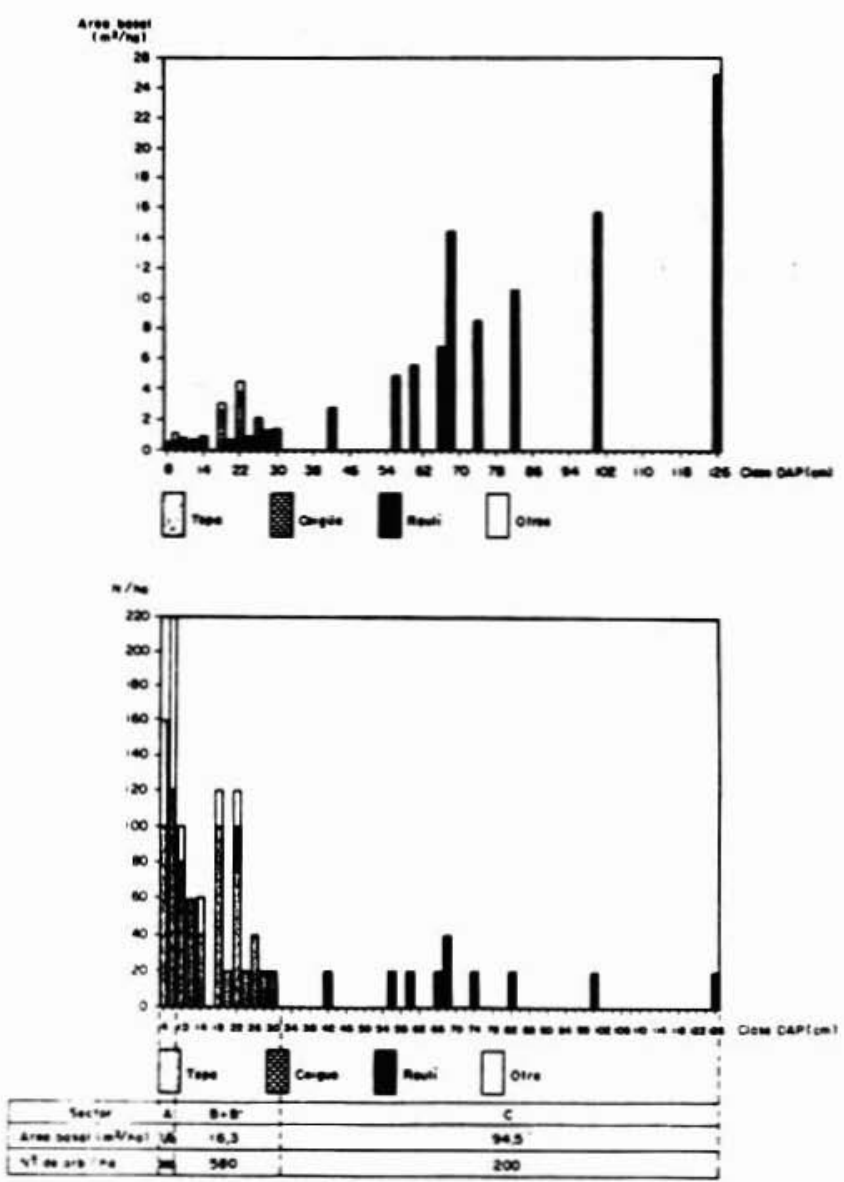

66 / Ciencia e Investigación Forestal 


\section{Discusión acerca de las intervenciones a renovales de tepa}

En muchos bosques del tipo forestal que incluyen la tepa como uno de sus componentes, la tradicional y continua extracción de los individuos del género Nothofagus ha transformado la composición de especies. De bosques claros se produjo el paso a rodales oscuros, debido al cambio en el estrato dominante de especies del género Nothofagus a especies de mayor tolerancia como la tepa.

Las dos situaciones descritas reflejan este fenómeno. En ambas, se encuentra un estrato dominante con un bajo número de individuos de grandes dimensiones, de roble en un caso y coigüe el otro. Debajo permanece un número suficiente de tepas para poder proyectar el rodal futuro.

Situaciones de este tipo dan la posibilidad de manejar el bosque en dos estratos.

El modelo de crecimiento utilizado, se construyó basado exclusivamente en tepas que estaban bajo un dosel superior de árboles del género Nothofagus. Por otro lado, las edades medias de las tepas para los dos rodales son bastante avanzadas. Ambos factores incidieron en que los crecimientos de las tepas de estos rodales, obtenidos por el modelo de crecimiento diametral, fluctúen entre $0,25 \cdot 0,30 \mathrm{~cm}$ anuales. Es de suponer, que con una mayor disponibilidad de luz y a menor edad, el crecimiento de esta especie supere ampliamente lo medido.

\section{Discusión sobre la proyección de renovales de raulí, roble, coigüe y tepa}

En la actualidad, gran parte de los renovales en Chile se encuentran dentro de la fase de desarrollo de crecimiento óptimo. En ésta, los árboles presentan un crecimiento vigoroso y los raleos favorecen en términos importantes a los individuos remanentes.

Muchos renovales se encuentran finalizando la fase de crecimiento óptimo, por lo que la capacidad de reaccionar frente a una liberación de los árboles es menor, aunque significativa.

Como información básica para estimar el potencial de crecimiento de un rodal antes de su intervención, debe conocerse su edad, composición y estructura. Esta información, junto a los antecedentes sobre demanda y precios de los productos a obtener, orientarán la decisión acerca de los tratamientos silviculturales a aplicar.

En relación al mercado de la madera de latifoliadas, se ha observado en los últimos años una baja en la oferta, debido a la eliminación de grandes reservas de estas especies en las zonas tropicales. En forma paralela ha aumentado la demanda por estas especies, producto del aumento de los ingresos en los países desarrollados.

Los precios de latifoliadas europeas han aumentado en respuesta a este fenómeno. Para Fagus sylvatica y Quercus sp., la tendencia de precios en relación a (la conífera) Picea se ilustra en la figura 11.

Especialmente notorio es el interés por madera de Encino la cual ha incrementado su valor de mercado en un $100 \%$ en relación a la de Picea en el transcurso de un período de 20 aก̃os.

Para madera de buena calidad de Fagus sylvatica los precios de rollizos a orilla de camino en la R.F.A. pueden llegar hasta US\$ 131, incluyendo diámetros sobre $20 \mathrm{~cm}$ (BURSCHEL y HUSS, 1987). Este precio podría tener validez como indicador para algunos Nothofagus, asumiendo cierta similitud entre éstos y los Fagus.

La tendencia del mercado de las latifoliadas, así como el alto rendimiento que se puede obtener en la mayoría de los renovales de Nothofagus, definen su objetivo de manejo. Este se traduce en la producción de madera de calidad con grandes diámetros para la cosecha final. 


\section{FIGURA 11}

EVOLUCION DE LOS PRECIOS PARA MADERA DE ENCINO Y FAGUS SYLVATICA EN RELACION A PICEA (FUENTE: HUSS, 1989)

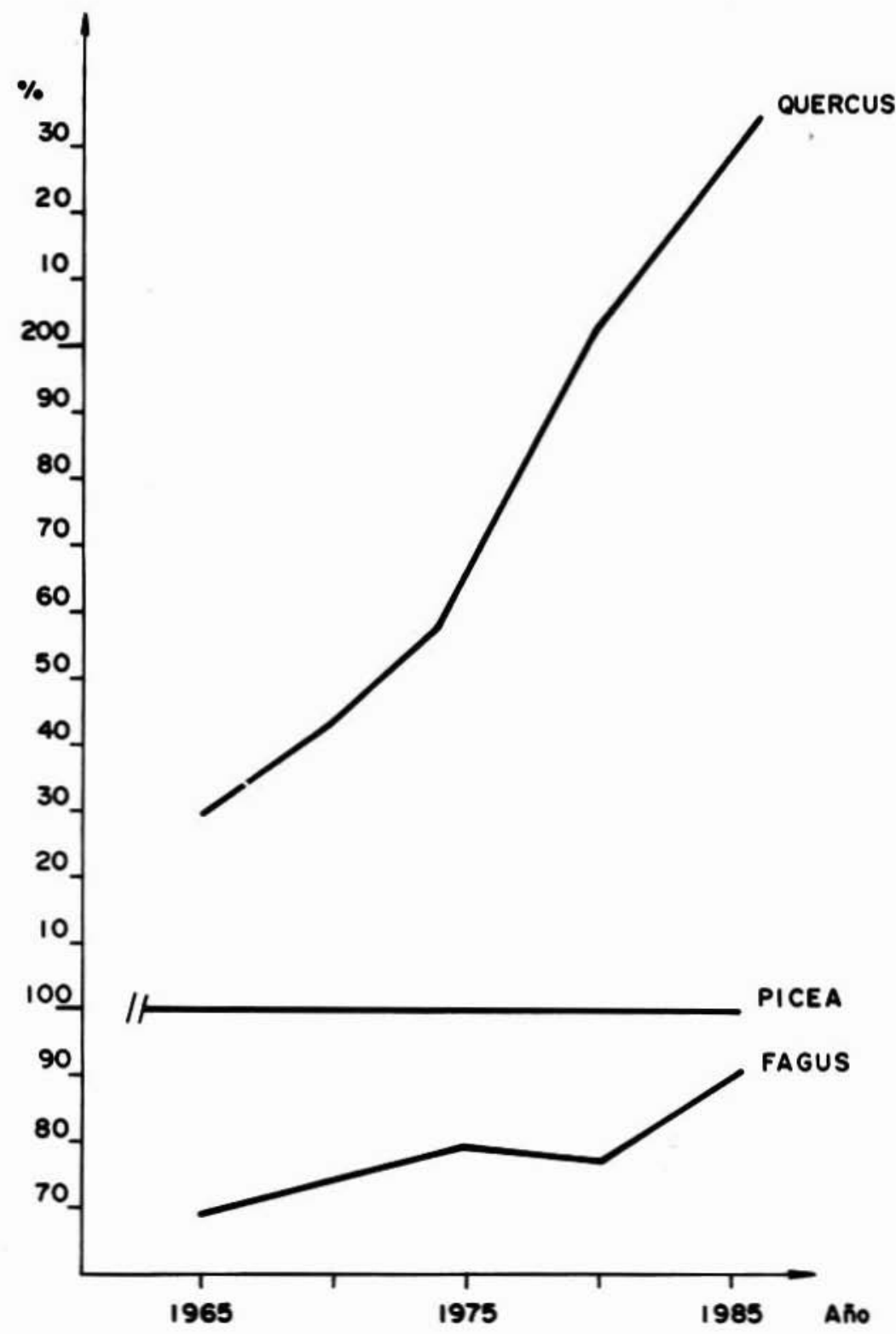

68 / Ciencia e Investigación Forestal 


\section{El desarrollo de los renovales}

Prácticamente, toda primera intervención a un renoval, implica la extracción de un volumen económicamente atractivo, que se puede traducir en madera aserrada, leña y chips. Sólo en situaciones de renoval muy joven debería realizarse un raleo a deshecho.

Intervenciones al estrato codominante y dominante, reduciendo el $\mathrm{AB}$ del estrato a proyectar en un 30-40\%, permiten extraer rollizos de dimensiones entre 15 y $40 \mathrm{~cm}$.

En el área estudiada el crecimiento observado en el raulí es superior al que presenta roble, coigüe y tepa. Las proyecciones realizadas demuestran que la edad del rodal y la calidad del sitio, tienen gran influencia en el rendimiento de los árboles. Especialmente notorio es el caso de coigüe, ya que se duplica el rendimiento obtenido en el sector de Los Hornos, en relación al obtenido en el sector de Truful (Cuadro 16).

Las tepas, que crecen bastante más lento que los Nothofagus, por lo general deberán actuar como especie acompañante por dos o más generaciones, aprovechando su gran tolerancia a la sombra:

Con las proyecciones realizadas, se logran diámetros medios entre $30 \mathrm{~cm}$ y $51 \mathrm{~cm}$ para los Nothofagus y de $28 \mathrm{~cm}$ a $31 \mathrm{~cm}$ para las tepas, en periodos que fluctúan entre 10 y 30 años (Cuadro 14).

En la mayoría de las situaciones, desde el inicio del manejo se generan ingresos, a pesar de que existen pérdidas de volumen por pudrición. Esto sucede especialmente cuando se trata de árboles de un estrato superior sobremaduro.

\section{CUADRO 16}

\section{PROYECCION DE RENOVALES DE RAULI, ROBLE, COIGUE y TEPA, MANEJADOS (Area Neltume)}

\begin{tabular}{|c|c|c|c|c|c|c|c|c|}
\hline \multirow[b]{2}{*}{ Espec. } & \multirow[b]{2}{*}{ Sector } & \multicolumn{4}{|c|}{ Extracción $\left(m^{3} / h a\right)$} & \multirow[b]{2}{*}{$\begin{array}{l}\text { Edad } \\
\text { al } \\
\text { año } 0\end{array}$} & \multirow[b]{2}{*}{$\begin{array}{l}\text { Años } \\
\text { a } \\
\text { proy. }\end{array}$} & \multirow[b]{2}{*}{$\begin{array}{c}\overline{\mathbf{D A}} \overline{\mathbf{A P}}(\mathrm{cm}) \\
\text { Objetivo }\end{array}$} \\
\hline & & $\begin{array}{c}\text { DAP } \\
\text { (cm) } \\
\text { (año 0) }\end{array}$ & Año 0 & $\begin{array}{l}\text { Interm. } \\
+ \text { cosecha }\end{array}$ & $\begin{array}{c}\text { Increm. } \\
\text { anual } \\
\left(\mathrm{m}^{3} / \mathrm{ha}\right)\end{array}$ & & & \\
\hline Raulí & $\begin{array}{l}\text { Los Hornos } \\
\text { Queb. Honda }\end{array}$ & $\begin{array}{l}24,8 \\
20,1\end{array}$ & $\begin{array}{c}190 \\
/\end{array}$ & $\begin{array}{r}647 \\
1.000\end{array}$ & $\begin{array}{l}22,4 \\
31,1\end{array}$ & $\begin{array}{l}36 \\
24\end{array}$ & $\begin{array}{l}20 \\
30\end{array}$ & $\begin{array}{l}39,2 \\
51,4\end{array}$ \\
\hline Roble & $\begin{array}{l}\text { Los Hornos } \\
\text { Fuy }\end{array}$ & $\begin{array}{l}20,9 \\
14,3\end{array}$ & $\begin{array}{r}48 \\
247\end{array}$ & $\begin{array}{l}384 \\
287\end{array}$ & $\begin{array}{l}14,7 \\
10,3\end{array}$ & $\begin{array}{l}41 \\
31\end{array}$ & $\begin{array}{l}15 \\
20\end{array}$ & $\begin{array}{l}31,8 \\
32,9\end{array}$ \\
\hline Coigüe & $\begin{array}{l}\text { Truful } \\
\text { Los Hornos }\end{array}$ & $\begin{array}{l}23,8 \\
40,5\end{array}$ & $\begin{array}{r}96 \\
134\end{array}$ & $\begin{array}{l}247 \\
433\end{array}$ & $\begin{array}{r}6,8 \\
14,6\end{array}$ & $\begin{array}{l}40 \\
46\end{array}$ & $\begin{array}{l}15 \\
10\end{array}$ & $\begin{array}{l}30,2 \\
49,5\end{array}$ \\
\hline Tepa & $\begin{array}{l}\text { Molco } \\
\text { Huilo }\end{array}$ & $\begin{array}{l}22,5 \\
18,2\end{array}$ & $\begin{array}{l}275 \\
844\end{array}$ & $\begin{array}{l}356 \\
232\end{array}$ & $\begin{array}{l}6,5 \\
4,8\end{array}$ & $\begin{array}{l}63 \\
81\end{array}$ & $\begin{array}{l}30 \\
30\end{array}$ & $\begin{array}{l}30,7 \\
27,9\end{array}$ \\
\hline
\end{tabular}




\section{La Silvicultura de los renovales}

La composición de especies y la estructura de los renovales es muy variada. En esta oportunidad se estudiaron las siguientes situaciones tipo:

A : Renoval puro de Nothofagus (monoespecífico y mixto).

B : Renoval mixto con Nothofagus en el estrato superior y especies tolerantes en el estrato inferior.

C : Renoval de tepa bajo dosel de Nothofagus sobremaduro (especialmente coigüe).

En términos generales los renovales de Nothofagus en la fase de crecimiento óptimo deberían manejarse liberando los individuos del estrato a proyectar de sus competidores más directos a nivel de los árboles dominantes y codominantes. No se deberá extraer más del $30-40 \%$ del área basal de dicho estrato. Con esto asegura una maximización del incremento volumétrico, la estabilidad del rodal frente al viento y se evitan daños por insolación (GROSSE, 1987).

Esta recomendación es válida para las situaciones A y B, donde el estrato superior está formado por Nothofagus. Si se trata de un renoval mixto con especies tolerantes valiosas bajo un dosel de Nothofagus (elegimos caso B), puede considerarse un manejo en dos estratos. En este caso, $\mathbf{y}$ debido a su lento crecimiento, las especies tolerantes se proyectan a través de dos o más generaciones de Nothofagus. Esto significa que la repoblación de Nothofagus después de la cosecha estará protegida por un dosel abierto de especies tolerantes.

Al finalizar la siguiente rotación, sería posible cosechar entonces el grupo de especies tolerantes e intolerantes.

Una tercera situación se presenta cuando el renoval está formado por especies tolerantes, creciendo bajo un dosel de Nothofagus sobremaduros de bajo valor comercial. En este caso deberá buscarse la proyección de las especies tolerantes a través de raleos y la eliminación de los individuos viejos del estrato superior. Esto último se logra a través de la extracción cuidadosa del dosel superior de manera de evitar daffos, o mediante el anillamiento en pie. Con este esquema se puede lograr un renoval puro de especies tolerantes.

Los esquemas analizados entregan posibilidades de manejo para renovales en uno y dos estratos, combinando especies de mayor y menor tolerancia a la sombra. La orientación que se le dé a los rodales, referida a la elección de las especies a proyectar depende de los objetivos del productor. Podrá elegir entre rodales puros de Nothofagus o mezclas de éstos con especies de mayor tolerancia.

Para un renoval entre 25 y 45 afos, el período de espera hasta realizar la cosecha final podrá fluctuar según el caso entre 10 y 30 af́os. Antes de realizar la explotación definitiva, es posible consolidar la generación futura, a través de plantación y regeneración natural bajo un dosel que se debe abrir entre 5 y 10 años antes de la cosecha definitiva. Con esto se garantiza una mayor sobrevivencia de los individuos regenerados.

Generaciones futuras con árboles del género Nothofagus, establecidas con plantas de buena calidad y con las intervenciones silvícolas requeridas durante su desarrollo, podrían llegar a su edad de corta entre los 30 y 40 años. Especies tolerantes deberán proyectarse durante dos generaciones de Nothofagus, es decir, aproximadamente entre 60 y 80 affos. 


\section{CONCLUSIONES}

A continuación se presentan las conclusiones más importantes referidas a la situación actual de los renovales del área de Neltume, su proyección y su silvicultura.

- Los renovales se encuentran dentro de la fase de desarrollo, definida como crecimiento óptimo, lo que permite incrementar su rendimiento a través de raleos.

- Los objetivos del manejo deberán estar orientados en base a la creciente demanda mundial por la madera de latifoliadas de calidad.

- Las rectitudes de fuste y forma cilíndrica observadas para rauli, roble, coigüe y tepa, permiten una proyección de los renovales estudiados dentro del mercado mundial de latifoliadas.

- $\quad \mathrm{Al}$ intervenir desde el comienzo de la fase de crecimiento óptimo se tendería a maximizar el rendimiento proyectado del rodal siendo factible llegar hasta un incremento volumétrico periódico de $30 \mathrm{~m}^{3}$ por ha al año. Para el caso del raulí se supera fácilmente un crecimiento diametral anual de un $\mathrm{cm}$.

De rodales del género Nothofagus manejados, se podrian esperar rotaciones entre 30 y 40 años.

Con intervenciones tardías dentro de la fase del crecimiento óptimo se logran incrementos diametrales cercanos a $1 \mathrm{~cm}$ por año en el raulí, llegándose a incrementos volumétricos periódicos sobre $20 \mathrm{~m}^{3}$ por ha al año.

- Para las áreas estudiadas el rendimiento estimado para raulí supera al de los robles y coigües.

- $\quad$ El crecimiento de tepa es menor al de las especies del género Nothofagus. Por este motivo su permanencia en el rodal puede llegar a dos rotaciones de Nothofagus. 


\section{REFERENCIAS BIBLIOGRAFICAS}

1. ALVAREZ, S. (1977): Programa de Análisis de tallo en Fortran IV. Archivo del INFOR.

2. BURSCHEL, P. y HUSS, J. (1987): Grundriss des Waldbaus. Paul Parey Hamburg - Berlin. 352 pp.

3. CUBILLOS, V. (1987): Modelo de crecimiento diametral para raulí. Ciencia e Investigación Forestal $\mathbf{N}^{\circ} 1$. INFOR - Chile. pág. 68-76.

4. CUBILLOS, V. (1988) a: Funciones de volumen y factor de forma para renovales de raulí. Ciencia e Inves. tigación Forestal $\mathrm{N}^{\circ}$ 3. INFOR - Chile. pág. 103-113.

5. CUBILLOS, V. (1988) b: Funciones de volumen y factor de forma para renovales de coigüe. Ciencia e Investigaciôn Forestal $\mathrm{N}^{\circ}$ 4. INFOR - Chile. pág. 62-68.

6. GROSSE, H. (1987): Desarrollo de renovales de raulí raleados. Ciencia e Investigación Forestal $\mathbf{N}^{\circ} 2$. INFOR - Chile. pág. 31-44.

7. GROSSE, H. y CUBILLOS, V. (1987): Estudio de renovales de raulí. (Informe INFOR - CORFO). $124 \mathrm{pp}$.

8. GROSSE, H., CUBILLOS, V., BOURKE, M. y URIARTE, A. (1988): Investigación Manejo Silvícola de diferentes tipos de Bosques Nativos. (Informe final INFOR - CORFO). 248 pp.

9. GROSSE, H. (1989): Antecedentes para el manejo de renovales de raulí. Corma $\mathrm{N}^{\circ}$ 206. Pág. 16-20.

10. HUSS, J. (1989): La investigación del bosque nativo. (Charla ofrecida en el INFOR Marzo de 1989). 


\title{
SELECCION DE PROCEDENCIAS DE PINO OREGON (Pseudotsuga menziesii (MIRB.) FRANCO) EN LA ZONA SUR DE CHILE
}

\begin{abstract}
RESUMEN
Se evalúan los resultados de tres ensayos de procedencias de Pino Oregón (Pseudotsuga menziesii (MIRB.) FRANCO) establecidos en la VIII y X Regiones del país.

A la edad de 15 años se encontraron diferencias estadísticamente significativas entre los volúmenes de las diferentes procedencias ensayadas. Los mejores resultados se obtuvieron en los dos ensayos establecidos en la zona costera de la VIII y X regiones. Las procedencias de la zona Noroeste de Estados Unidos, especialmente las del estado de Washington son las que presentan los mejores crecimientos.
\end{abstract}

\begin{abstract}
A Douglas fir (Pseudotsuga menziesii (Mirb.) Franco) provenance trial established in three Southern locations of Chile was assessed.

Fifteen years after the establishment, the total volumes are compared. ANOVA indicates the existence of estatistically significant differences among the tested provenances. The best results were achieved in coastal experimental sites. The north western provenances (Washington State) showed the best growth rates, among the 10 seed origins tested.
\end{abstract}

(*) Ingeniero Forestal, División Silvicultura, Instituto Forestal, Huérfanos 554 - Piso 4 - Santiago - Chile.

(**) Ingeniero Forestal, División Silvicultura, Instituto Forestal, Huérfanos 554 - Piso 4 - Santiago - Chile. 


\section{INTRODUCCION}

Dentro del contexto forestal mundial, la especie Pino Oregón (Pseudotsuga menziesii (MIRB.) FRANCO) constituye una de las de mayor importancia económica. En su lugar de origen, Norteamérica, se distribuye ampliamente tanto en latitud como en longitud y ocupa una superficie de cerca de 13 millones de ha, con un volumen aproximado a 2.650 millones de metros cúbicos en pie. (USDA, 1982).

Debido a la gran capacidad de adaptarse y a las excelentes características de la madera, es una especie que se ha constituido en un recurso muy importante a nivel mundial. (UN, 1985).

En nuestro país esta especie fue introducida hace más de 90 años. Recién en 1928 , se plantó en forma masiva en la zona de Traiguén. Dentro de las especies coníferas introducidas, el Pino Oregón es la segunda en importancia después de Pino Radiata. En efecto, hoy día ocupa una superficie de aproximadamente $11.000 \mathrm{ha}$, que se distribuyen preferentemente entre la IX y X Regiones (INFOR, 1987).

Previo a considerar esta especie en un programa de forestación, es de suma importancia analizar el origen de la semilla, ya que la procedencia es determinante en el éxito del establecimiento y productividad de las plantaciones.

En este trabajo se analizan la adaptabilidad y el crecimiento de 10 procedencias de Pino Oregón, establecidas por INFOR en 1968 en tres lugares de ensayo, situados en la VIII y la $\mathrm{X}$ Región.

\section{MATERIAL Y METODO}

\section{Lugares de Procedencia}

Las procedencias ensayadas provienen, en su mayoría, de las poblaciones costeras del Noroeste de los Estados Unidos y de la provincia de British Columbia, en Canadá. Sólo una corresponde a las poblaciones interiores, ya que proviene de Coconino, Arizona. En la Figura 1 se presenta el área de distribución natural del Pino Oregón excluyendo las zonas de México, (READ Y SPRACKLING, 1976) y se indican las procedencias ensayadas.

La ubicación geográfica se determinó según datos del proveedor de semillas (Silvaseed Company, Roy. Washington 98580 , USA). La procedencia exacta dentro de la zona de Coconino, Arizona, se estimó de acuerdo al área de mayor ocurrencia natural del Pino Oregón.

En el Cuadro 1 se detallan los antecedentes de ubicación de los lugares de procedencia y los datos climáticos más relevantes. Estos se obtuvieron de las estaciones metereológicas más cercanas a las zonas de origen de la semilla. (U.S. DEPT. OF COMMERCE, 1959-1965). 
FIGURA 1

AREA DE DISTRIBUCION NATURAL DEL PINO OREGON (*)

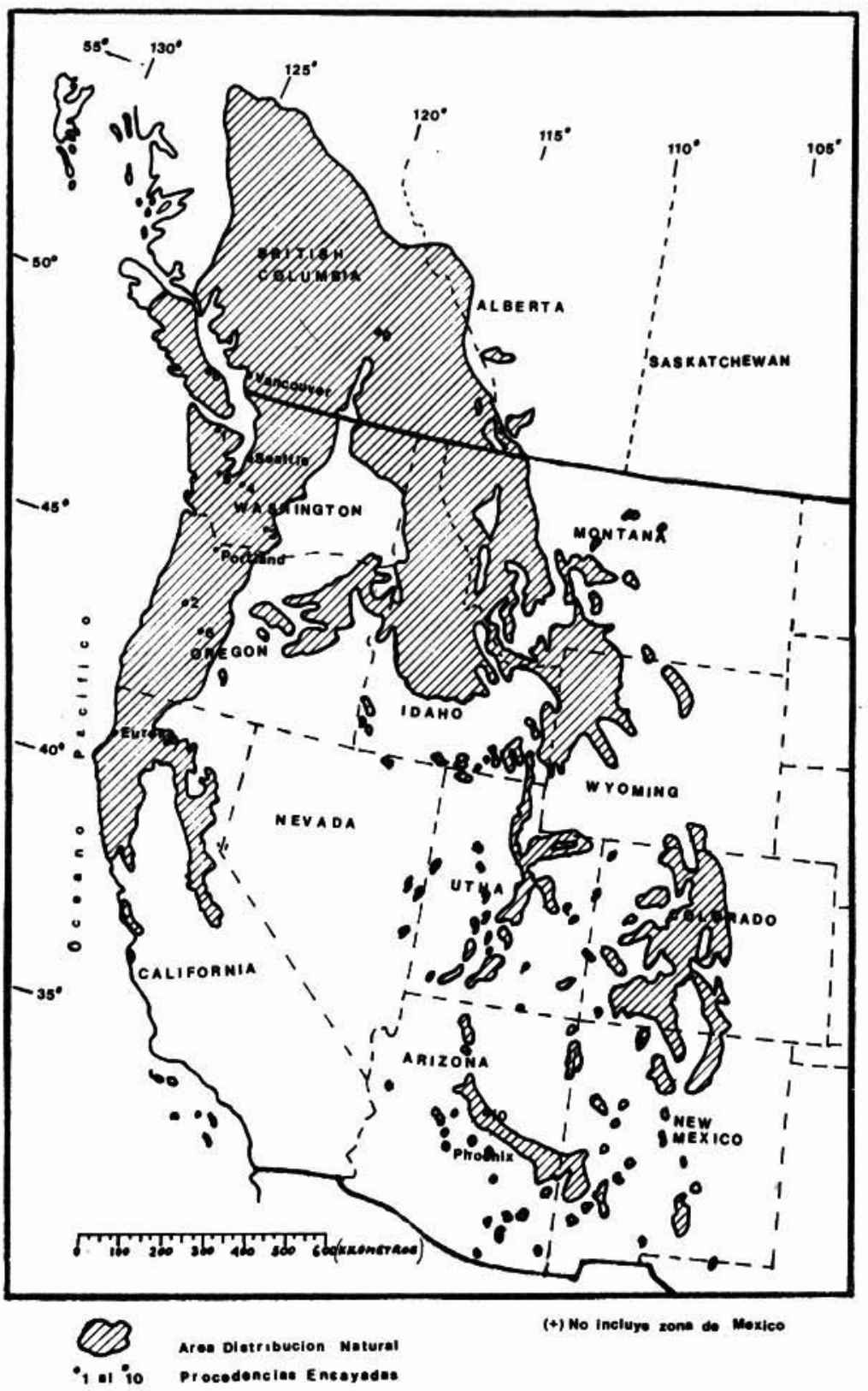

(*) Read and Sprackling, 1976 